\title{
Identification and expression analysis of the DREB transcription factor family in pineapple (Ananas comosus (L.) Merr.)
}

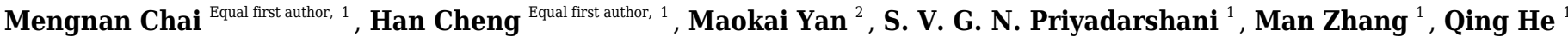

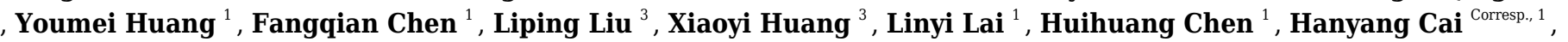 \\ Yuan Qin Corresp. 1, 2,3 \\ ${ }^{1}$ State Key Lab of Ecological Pest Control for Fujian and Taiwan Crops; Key Lab of Genetics, Breeding and Multiple Utilization of Crops, Ministry of \\ Education; Fujian Provincial Key Lab of Haixia Applied Plant Systems Biology, Fujian Agriculture and Forestry University, Fuzhou, Fujian Province, China \\ 2 State Key Laboratory for Conservation and Utilization of Subtropical Agro-Bioresources, Guangxi Key Lab of Sugarcane Biology, College of Agriculture, \\ Guangxi University, Nanning, Guangxi Province, China \\ 3 College of Life Sciences, Fujian Agriculture and Forestry University, Fuzhou, Fujian Province, China \\ Corresponding Authors: Hanyang Cai, Yuan Qin \\ Email address: 907591658@qq.com, yuanqin@fafu.edu.cn
}

Background: Dehydration responsive element-binding (DREB) transcription factors play a crucial role in plant growth, development, and stress responses. Although DREB genes have been characterized in many plant species, genome-wide identification of the DREB gene family has not yet been reported in pineapple (Ananas comosus (L.) Merr.).

Results: Using comprehensive genome-wide screening, we identified 20 AcoDREB genes on 14 chromosomes. These were categorized into five subgroups. AcoDREBs within a group had similar gene structures and domain compositions. Using gene structure analysis, we showed that most ACODREB genes (75\%) lacked introns, and that the promoter regions of all 20 AcoDREB genes had at least one stress response-related cis-element. We identified four genes with high expression levels and six genes with low expression levels in all analyzed tissues. We detected expression changes under abiotic stress for eight selected ACODREB genes.

Conclusions: This report presents the first genome-wide analysis of the DREB transcription factor family in pineapple. Our results provide preliminary data for future functional analysis of $A C O D R E B$ genes in pineapple, and useful information for developing new pineapple varieties with key agronomic traits such as stress tolerance. 
1 Identification and expression analysis of the DREB

2 transcription factor family in pineapple (Ananas

3 comosus (L.) Merr.)

4

5

6

7

8

9

10

11

12

13

14

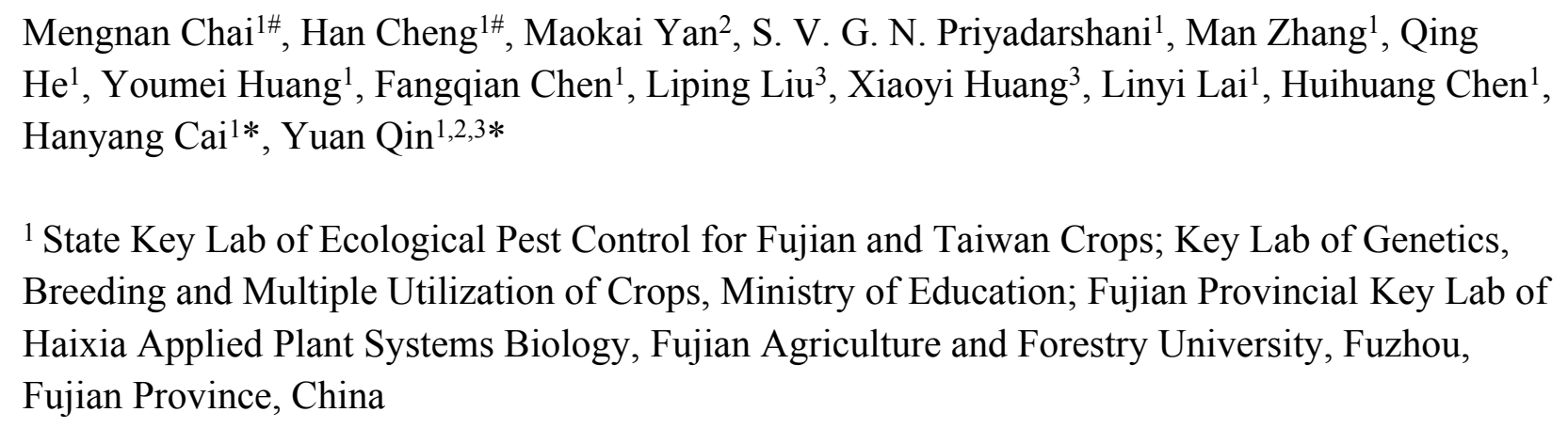

${ }^{2}$ State Key Laboratory for Conservation and Utilization of Subtropical Agro-Bioresources, Guangxi Key Lab of Sugarcane Biology, College of Agriculture, Guangxi University, Nanning, Guangxi, China

${ }^{3}$ College of Life Sciences, Fujian Agriculture and Forestry University, Fuzhou, Fujian Province, China

"These authors contributed equally.

Corresponding Author:

Hanyang Cai ${ }^{1}$

No. 15, Shangxiadian Road, Fuzhou, Fujian Province 350002, China

Email address:907591658@qq.com

Yuan Qin ${ }^{1,2,3}$

No. 15, Shangxiadian Road, Fuzhou, Fujian Province 350002, China

Email address: yuanqin@fafu.edu.cn

\section{ABSTRACT}

Background: Dehydration responsive element-binding (DREB) transcription factors play a crucial role in plant growth, development, and stress responses. Although DREB genes have been characterized in many plant species, genome-wide identification of the DREB gene family has not yet been reported in pineapple (Ananas comosus (L.) Merr.).

Results: Using comprehensive genome-wide screening, we identified 20 AcoDREB genes on 14 chromosomes. These were categorized into five subgroups. AcoDREBs within a group had similar gene structures and domain compositions. Using gene structure analysis, we showed that 
most $A c o D R E B$ genes (75\%) lacked introns, and that the promoter regions of all 20 AcoDREB genes had at least one stress response-related cis-element. We identified four genes with high expression levels and six genes with low expression levels in all analyzed tissues. We detected expression changes under abiotic stress for eight selected AcoDREB genes.

Conclusions: This report presents the first genome-wide analysis of the DREB transcription factor family in pineapple. Our results provide preliminary data for future functional analysis of $A c o D R E B$ genes in pineapple, and useful information for developing new pineapple varieties with key agronomic traits such as stress tolerance.

Keywords: pineapple, DREB transcription factors, phylogenetic analysis, expression profiles

\section{INTRODUCTION}

Abiotic stress, such as salinity, drought, and high or low temperatures, severely affects the growth and development of plants. To adapt to these stressors, plants have evolved complex signal transduction pathways and response mechanisms that are induced by specific functional and regulatory proteins. Functional proteins that respond to stress include membrane proteins (transporters and water channel proteins), osmolyte biosynthesis enzymes (to produce proline, betaine, soluble sugars, etc.), detoxification enzymes (catalase, superoxide dismutase, ascorbate peroxidase, glutathione $S$-transferase, etc.), and other proteins that help protect macromolecules (LEA protein, osmotin, antifreeze proteins, mRNA binding protein, etc.). Regulatory proteins that respond to stress include transcription factors (bZIP, MYC, MYB, DREB, etc.), protein kinases (receptor protein kinase, MAP kinase, CDP kinase, transcription-regulation protein kinase, etc.), and proteinases (phospholipase C, phosphoesterases, etc.) (Agarwal et al. 2006). Among the regulatory proteins, transcription factors (TFs) play pivotal roles in abiotic stress responses. Specifically, they activate or repress the expression of stress-response genes by recognizing and binding to cis-elements in the promoters of their targets (Golldack et al. 2011; Malhotra \& Sowdhamini 2014; Agarwal et al. 2017). They are the main targets of genetic engineering for enhancing stress tolerance in crop plants (Century et al. 2008).

Dehydration response element-binding (DREB) TFs enhance plant tolerance to abiotic stresses by specifically binding dehydration response element/C-repeat (DRE/CRT) cis-elements to control downstream gene expression. The DREB TF family belongs to the APETALA2/ETHYLENE-RESPONSIVE FACTOR (AP2/ERF) superfamily of TFs. The AP2/ERF superfamily is characterized by the AP2 domain, which is 60-70 amino acids long, and contains two conserved sequence blocks, the YRG element and the RAYD element. The YRG element is 19-22 amino acids long and contains the conserved YRG motif, which may confer DNA-binding specificity to the AP2 protein. The RAYD element has a conserved core region that can form an amphipathic $\alpha$-helix in the AP2 domain (Okamuro et al. 1997). The AP2 domain of the DREB subfamily differs at specific amino acid sites from that of other 
79

80

81

82

83

84

85

86

87

88

89

90

91

92

93

94

95

96

97

98

99

100

101

102

103

104

105

106

107

108

109

110

111

112

113

114

115

116

117

subfamilies. These include the valine (Val14) and glutamine (Glu19) residues, which are conserved in the DREB subfamily (Sakuma et al. 2002).

The DREB subfamily members in Arabidopsis thaliana can be classified into six groups, named A-1 to A-6, or DREB1 to DREB6 (Sakuma et al. 2002). Of these, the TFs belonging to A-1 and A-2 are functionally well characterized. The first identified $D R E B$ gene was the A-1 member $A t C B F 1$, which is strongly induced by low temperature. In addition, AtDREB1A and AtDREB IC positively regulate low-temperature stress responses (Jaglo-Ottosen et al. 1998; Liu et al. 1998). $S w D R E B 1$ from sweet potato (Ipomoea batatas) is involved in the response to low temperature (Kim et al. 2008). Heterologous overexpression of zoysia grass (Zoysia japonica) ZjDREB1.4 in Arabidopsis enhanced tolerance to high and freezing temperature stresses without obvious growth inhibition (Feng et al. 2019). In rice (Oryza sativa), the interaction of OsDREB1A, OsDREB1B, and OsDREB1C with the GCC box enhanced the cold tolerance of the plants (Donde et al. 2019). Thus, DREB1 TFs are mainly associated with cold stress regulation.

By contrast, DREB2 is mainly associated with drought and salinity tolerance (Liu et al. 1998). AtDREB2A and AtDREB2B, the first reported A-2 members, are induced by dehydration and salinity (Sakuma et al. 2002). Overexpression of soybean (Glycine max) GmDREB2 in Arabidopsis enhanced salinity tolerance without growth retardation (Chen et al. 2007). In sugarcane (Saccharum spp. Hybrid), heterologous overexpression of EaDREB2 enhanced the tolerance of plants to drought and salinity stress (Augustine et al. 2015).

In contrast to A-1 and A-2 proteins, the functions of A-3 to A-6 members are only beginning to be uncovered. The maize (Zea mays) A-4 subgroup gene ZmDREB4.1 was associated with the negative regulation of plant growth and development (Li et al. 2018). A novel A-5 subgroup gene from desert moss (Syntrichia caninervis), ScDREB8, enhanced the salt tolerance of Arabidopsis seedlings by up-regulating the expression of stress-related genes (Liang et al. 2017). CmDREB6 belongs to the A-6 subgroup, and its overexpression enhanced the tolerance of chrysanthemum (Chrysanthemum morifolium) to heat stress (Du et al. 2018).

Pineapple (Ananas comosus (L.) Merr.), the third most important tropical fruit in world production, is widely grown in tropical and subtropical regions (Moyle et al. 2005). The crop has high economic value, and pineapple cultivation is of great significance to the development of local agriculture. However, the changes in global climate have underscored how different abiotic and biotic stresses critically affect the growth of pineapple (Mittler 2006; Ray et al. 2013). Pineapples are damaged under severe drought and high temperature. Low temperatures diminish growth. Biotic stressors such as pests, diseases, and weeds also lead to significant yield loss (Lobo \& Paull 2016). 
118 DREB family genes have been identified in A. thaliana (Hwang et al. 2012), perennial ryegrass

119

120

121

122

123

124

125

126

127

128

129

130

131

132

133

134

135

136

137

138

139

140

141

142

143

144

145

146

147

148

149

150

151

152

153

154

155

156

(Xiong \& Fei 2006), Triticum L. (Mondini et al. 2015), Dendranthema (Yang et al. 2009), Zea mays (Qin et al. 2007), and Oryza sativa L. (Cui et al. 2011; Gumi et al. 2018; Matsukura et al. 2010). According to previous research in several plant species, most $D R E B$ genes respond to various stress conditions. However, $D R E B$ genes have never been reported in pineapple. Therefore, our analysis focused on the identification of $A c o D R E B$ genes as well as the characteristics of the encoded DREB TFs. In this study, we identified 20 AcoDREB genes belonging to five subgroups and analyzed their gene and protein structures, protein motifs, chromosomal distribution, and expression profiles. Our results provide a relatively complete profile of the pineapple $D R E B$ gene family. This may aid further functional analysis of each member, and facilitate the improvement of pineapple varieties via gene-transfer techniques, to confer tolerance to abiotic and biotic stresses (Priyadarshani et al. 2019).

\section{MATERIALS \& METHODS}

\section{Identification of DREB family members in pineapple}

DREB amino acid sequences from Oryza sativa and Arabidopsis thaliana were obtained from the Rice Genome Annotation Project (RGAP, http://rice.plantbiology.msu.edu/index.shtml) (Kawahara et al. 2013) and The Arabidopsis Information Resource (TAIR, http://www.arabidopsis.org) (Berardini et al. 2015), respectively. The DREB sequences from Arabidopsis were used as search queries in BLAST-P against the pineapple genome. The AP2 (PF00847) domain was downloaded and used as a query to perform a HMMER search with default parameters (https://www.ebi.ac.uk/Tools/hmmer/search/phmmer). HMMER is a software package that uses profile hidden Markov Models (HMM) to identify conserved domains (Ming et al. 2015). Redundant sequences were eliminated and the Simple Modular Architecture Research Tool (SMART, http://smart.embl-heidelberg.de/) (Letunic \& Bork 2018) was used to verify the existence and completeness of the core domain within the identified sequences. The sequences that met these criteria were used for phylogenetic analysis.

\section{Protein characteristics and chromosomal localization}

For each of the putative $A c o D R E B$ genes, the gene length, amino acid number, coding sequence (CDS) length, and chromosome position were collected from the Pineapple Genomics Database (PGD, http://pineapple.angiosperms.org/pineapple/html/index.html) (Xu et al. 2018). The molecular weights and isoelectric points of the putative proteins were predicted using the ExPASy proteomics server (http://expasy.org/) (Gasteiger et al. 2003). Based on the start positions of the genes and the lengths of the corresponding chromosomes, MapChart (Voorrips 2002) was used to visualize the $20 A c o D R E B$ genes that were mapped onto the 25 pineapple chromosomes and scaffold sequences.

\section{Cis-element analysis of $A c o D R E B$ gene promoters}


157 The $2 \mathrm{~kb}$ upstream sequences of the $A c o D R E B$ genes were retrieved from the Pineapple 158 Genomics Database and submitted to Plant Cis-Acting Regulatory Element (PlantCARE, $159 \mathrm{http} / / /$ bioinformatics.psb.ugent.be/webtools/plantcare/html/) (Lescot et al. 2002) to detect the

160

161

162

163

164

165

166

167

168

169

170

171

172

173

174

175

176

177

178

179

180

181

182

183

184

185

186

187

188

189

190

191

192

193

194

195 presence of the following six regulatory elements (Sazegari et al. 2015): abscisic acid (ABA)responsive elements (ABREs; ACGTG/TC), which are involved in ABA responsiveness (Yamaguchi-Shinozaki \& Shinozaki 1993); dehydration-responsive elements (DREs; A/GCCGAC), which are involved in plant responses to dehydration, low temperature, and salt stress (Narusaka et al. 2003); low temperature-responsive elements (LTREs; CCGAA), which are involved in low-temperature responses (Roy Choudhury et al. 2008); TC-rich repeats (G/ATTCTCT), which are involved in defense and stress responses (Diaz-De-Leon et al. 1993); W-boxes (TGACC/T), which are the binding site of WRKY TFs in defense responses (Jiang et al. 2017); and MBS (TAACTG), or MYB binding sites, which are involved in droughtinducibility (Urao et al. 1993).

\section{Sequence alignment and phylogenetic analysis}

The CDS of the AcoDREB genes were obtained from the Pineapple Genomics Database and imported into DNAMAN Version 9 for sequence alignment (Wang 2016). The phylogenetic tree was constructed in IQ tree using the maximum likelihood method (Chernomor et al. 2016; Nguyen et al. 2015). For this analysis, the parameters were set to default, except for the ultrafast bootstrap option, which was set to $\mathrm{n}=1000$ (Hoang et al. 2018), after performing multiple sequence alignments in MUSCLE 3.7 (Edgar 2004) using default parameters. To validate the maximum likelihood (ML) results, the neighbor-joining (NJ) method was used to construct a tree using MEGA7 (Kumar et al. 2016).

\section{Gene structure analysis and conserved motif identification}

The DREB gene structures, including the numbers and positions of exons and introns, were determined using the Gene Structure Display Server (GSDS, http://gsds.cbi.pku.edu.cn/) (Guo et al. 2007). Multiple EM for Motif Elicitation (MEME, http://meme-suite.org/tools/meme) was used to analyze the amino acid sequences of the 20 AcoDREBs; the maximum number of motifs was set to 10, and default parameters were used (Bailey et al. 2009).

\section{Plant material and growth conditions}

The pineapple (Ananas comosus) variety MD2 was provided by the Qin Lab (Haixia Institute of Science and Technology, Fujian Agriculture and Forestry University, Fujian, China)

(Priyadarshani et al. 2018). Plants were grown on a soil mixture containing 2:1 (v/v) peat moss:perlite, in plastic pots in a greenhouse under the following conditions: $30^{\circ} \mathrm{C}, 60-70 \mu \mathrm{mol}$ photons $\mathrm{m}^{-1} \mathrm{~s}^{-1}$ light intensity, $70 \%$ humidity, and a 16-h light/8-h dark photoperiod.

\section{RNA-Seq of different pineapple tissues}


196 We used an RNA extraction kit (Omega Bio-Tek, Shanghai, China) to extract total RNA from

197 the following tissues: calyx, gynoecium, ovule, petal, and stamen. The tissues were collected

198 according to previously described methods (Chen et al. 2017). The NEBNext Ultra RNA Library

199 Prep Kit for Illumina was used to prepare libraries prior to sequencing. RNA-Seq data for root,

200 leaf, leaf base, leaf tip, flower, and fruit at different development stages were collected from the

201 Pineapple Genomics Database (Ming et al. 2015). Using TopHat v2.1.1 (Trapnell et al. 2012)

202 with default parameters, the trimmed paired-end reads of all samples were aligned to the

203 pineapple genome. Cufflinks v2.2.1 and Cuffdiff v2.2.1 were used to estimate the Fragments Per

204 Kilobase of exon model per Million mapped (FPKM) values. The heatmap showing the

$205 A c o D R E B$ gene expression profile was generated using the pheatmap package in R (Galili et al.

206 2018).

207

208

209

210

211

212

213

214

215

216

217

218

219

220

221

222

223

224

225

226

\section{Stress treatments}

One-month-old plants in rooting medium were used as the planting material for the stress treatment analyses. Uniform tissue-cultured seedlings were obtained from the Qin Lab (Priyadarshani et al. 2018). Seedlings were subjected to the following stress treatments: low temperature $\left(4^{\circ} \mathrm{C}\right)$, high temperature $\left(45^{\circ} \mathrm{C}\right)$, drought $(350 \mathrm{mM}$ mannitol), and high salt (150 $\mathrm{mM} \mathrm{NaCl}$ ). Root and leaf tissues were collected at 6, 12, 24, and 48 hours after treatment. Seedlings that were not subjected to any of the stress treatments were used as controls. The collected samples were immediately stored in liquid nitrogen prior to total RNA extraction (Rahman et al. 2017).

\section{Quantitative real-time PCR and data analysis}

Total RNA was extracted using the Plant RNA Kit (Omega Bio-Tek, Shanghai, China) according to the manufacturer's instructions. The RNA concentrations ranged from 100 to $500 \mathrm{ng} / \mu \mathrm{l}$, and the $\mathrm{OD}_{260} / \mathrm{OD}_{280}$ ratios ranged from 1.8 to 2.0. According to the supplier's instructions for AMV reverse transcriptase (Takara Bio, Beijing, China), $1 \mu \mathrm{g}$ of purified total RNA was reverse transcribed into cDNA in a total reaction volume of $20 \mu \mathrm{l}$ (Cai et al. 2019). To quantify the relative transcript levels of selected $D R E B$ genes, real-time PCR was performed with genespecific primers on the Bio-Rad Real-time PCR system (Foster City, CA, USA) according to the manufacturer's instructions. The gene-specific primers used for this analysis are listed in

227 Supplementary Table S1. The PCR program used the following conditions: $95^{\circ} \mathrm{C}$ for $30 \mathrm{~s}, 40$

228 cycles of $95{ }^{\circ} \mathrm{C}$ for $5 \mathrm{~s}$ and $60{ }^{\circ} \mathrm{C}$ for $34 \mathrm{~s}$, and $95^{\circ} \mathrm{C}$ for $15 \mathrm{~s}$. For all tested genes, three technical 229 replicates and at least three independent biological replicates were used (Cai et al. 2017; Zhang 230 et al. 2018). Relative expression was calculated using the $2^{-\Delta \Delta C t}$ method (Century et al. 2008). 231 Data were analyzed using one-way analysis of variance (ANOVA). Significant differences 232 between treatments and controls are indicated by asterisks (* indicates a p-value $<0.05$ and ** 233 indicates a p-value $<0.01)$ (Table S2).

234

235

\section{RESULTS}


236 Genome-wide identification and chromosomal locations of pineapple $D R E B$ genes

237 Using Arabidopsis DREB amino acid sequences as search queries in BLAST, 20 DREB amino

238 acid sequences were obtained from the pineapple proteome. The corresponding genes were

239 named AcoDREB1 to AcoDREB20 (Table S3), and the amino acid sequences are listed in Table

240 S4. Table 1 lists the following information for the 20 genes: gene name, gene ID, nucleotide and

241 amino acid lengths, and the predicted isoelectric point ( $\mathrm{pI})$ and molecular weight $(\mathrm{Mw})$ of the

242 encoded protein. The protein lengths ranged from 149 (AcoDREB13) to 463 (AcoDREB20)

243 amino acids, and the CDS lengths ranged from 450 (AcoDREB13) to 1392 (AcoDREB20) bp.

244 The predicted protein molecular weights ranged from 16316.44 (AcoDREB13) to 49311.65

245 (AcoDREB20) Da, and the predicted isoelectric points ranged from 4.71 (AcoDREB 10) to 9.68

246 (AcoDREB07) (Table S5). The 20 AcoDREB genes mapped to 14 pineapple chromosomes (Fig.

247 1), with three genes on Chr2 and two genes each on Chr3, Chr5, Chr6, and Chr17. Nine other

248 chromosomes each contained one $A c o D R E B$ gene.

249

250

251

252

253

254

255

256

257

258

259

260

261

262

Multiple sequence alignment and phylogenetic analysis of the DREB family

Multiple sequence alignment of the AcoDREB AP2 domains indicated that the domain was highly conserved among the 20 AcoDREBs, and that it displayed characteristics typical of other DREB proteins (Fig. 2). Beyond the conserved YRG and RAYD motifs, all 20 AP2 domain sequences contained a Val residue at position 14 (Val14), and 11 of them had a Glu residue at position 19 (Glu19). Val14 is more important than Glu19 for the binding of DREB to the DRE cis-acting elements (Sakuma et al. 2002).

To determine the phylogenetic relationships between the DREB family members, we constructed a multi-species phylogenetic tree using the full-length amino acid sequences of DREBs from pineapple, Arabidopsis (Table S6), and rice (Table S7). In Fig. 3, AT3G57600 and AT2G40220 (red frame) belong to the Arabidopsis subgroups A-2 and A-3, respectively. Because none of the pineapple $D R E B$ genes were homologous to the A-3 subgroup, we divided the AcoDREBs into five subgroups, I to V (Fig. 3). group I included AcoDREB01, 02, and 03, group II included AcoDREB04, 05, 06, and 19, group III included AcoDREB07, 08, 09, and 10, group IV included

265

266

267 AcoDREB11, 12, 13, 14, and 15, and group V included AcoDREB16, 17, 18, and 20.

268

\section{Stress-related cis-elements in $A c o D R E B$ promoters}

Because of the potential involvement of $A c o D R E B$ genes in stress responses, we investigated the

269 distribution of stress-related conserved cis-elements in their promoter regions $(2 \mathrm{~kb}$ region

270

271 upstream of the transcription start site) using PlantCARE (Table S8). The data for six abiotic

272

273 stress response elements, ABRE, DRE, LTRE, TC-rich repeat, MBS, and W-box, are shown in Fig. 4. All of the AcoDREB genes possessed at least one kind of cis-acting regulatory element, indicating that $A c o D R E B$ expression is associated with abiotic stress. Nine AcoDREBs had one

274 or more LTREs, which are associated with the response to low-temperature conditions. Sixteen

275 AcoDREBs contained between one and eight ABA-responsive elements, and only AcoDREB09, 
276 12, and 17 had the TC-rich repeat element. Seven AcoDREBs had the MBS element, while W-

277 boxes and DREs both occurred in ten AcoDREBs. Overall, the results of the cis-element analysis

278 indicate that $A c o D R E B$ genes can respond to different kinds of abiotic stresses.

279

280

281

\section{AcoDREB gene structure and conserved motifs in the encoded proteins}

282

Structural diversity is very common among duplicated genes, and may result in the evolution of

283 functionally distinct paralogs. To analyze the $A c o D R E B$ gene structures, exon, and intron

284 numbers and positions were determined by comparing the full-length cDNA sequences to the corresponding genomic DNA sequences (Fig. 5). Seventy five percent of the AcoDREB genes $(15 / 20)$ lacked introns. Four genes (AcoDREB18, 04, 19, and 13) had one intron each, and AcoDREB05 had three introns. Interestingly, the members of group II differed in terms of exon and intron number as well as UTR length, which suggests that these four paralogs may have different roles in pineapple growth and development.

289

290

291

As shown in Fig. 6, the distribution of the motifs among AcoDREB proteins was relatively

292 conserved. Motifs 1, 2, and 3 were present in all genes, but the motifs in different subgroups indicated some degree of divergence among them. For example, the three members in subgroup I contained motifs 4,5 , and 9 in addition to motifs 1,2 , and 3 . Motif 7 was only present in two of the subgroup III proteins (AcoDREB07 and AcoDREB08), and motif 4 was only present in AcoDREB05 of subgroup II. Generally, members within the same subgroup had similar motif compositions, indicating that they may perform similar functions (Fig. S1).

\section{AcoDREB gene expression profiles in different tissues at different developmental stages}

The different stages of the reproductive organs were defined according to previous studies (Azam

301 et al. 2018; Su et al. 2017). We used transcriptome sequencing data to analyze the expression patterns of the 20 AcoDREB genes in nine different tissues: root, leaf, flower, fruit, gynoecium, stamen, petal, calyx, and ovule (Fig. 7; Table S9). We also used quantitative real-time PCR (qRT-PCR) to verify the results of the RNA-seq. All AcoDREB genes, except four that had low levels of expression (AcoDREB04, 07, 08, and 13), were selected for qRT-PCR analysis in seven tissues. The results obtained were consistent with the RNA-Seq expression data of these genes (Fig. 8, Table S10).

Clustering analysis of the expression patterns of the 20 genes divided them into four clusters (Fig. 7). Of the six genes in cluster I, four (AcoDREB05, 16, 17, and 20) were highly expressed in all tissues, indicating that they may have important roles throughout plant growth. The expression level of AcoDREB09 was lower in stamens than in other tissues, and AcoDREB 19 had the lowest expression in roots, suggesting that these particular cluster I genes may not be critical for the development of these respective tissues. The six genes in cluster III (AcoDREB02, 04, 07, 08,10 , and 13 ) had very low expression levels in all tissues, suggesting that these genes might only be expressed under specific conditions. Most of the genes in clusters II and IV had tissue- or 
316 stage-specific expression patterns. For example, AcoDREB01 and AcoDREB15 had higher 317 expression in calyxes, suggesting that they may have a positive role in floral organ development.

318 The higher expression of AcoDREB06 in stage 6 stamens suggests a potential link to stamen 319 maturity. AcoDREB 18 was highly expressed during stamen development. AcoDREB11 was expressed in the ovule, stamen, and gynoecium tissues, suggesting this gene may function widely

321 during gametophyte development. AcoDREBO3 was highly expressed in the root, calyx, petal, 322 and gynoecium.

323

324

325

326

327

328

329

330

331

332

333

334

335

336

337

338

339

340

341

342

343

344

345

346

347

348

349

350

351

352

353

\section{AcoDREB gene expression under abiotic stress}

We analyzed $A c o D R E B$ gene expression under various abiotic stress conditions, including salt, drought, cold, and heat. Specifically, we examined the expression patterns of eight AcoDREB genes (AcoDREB01, 03, 06, 09, 11, 14, 18, and 19) in the MD2 variety of pineapple using qRTPCR with three biological and three technical replicates (Fig. 9, Table S2). Under all stress conditions, the relative transcript levels of the AcoDREB genes fluctuated during the 48-hour analysis period.

We subjected pineapple plants to salt stress by treating them with $150 \mathrm{mM} \mathrm{NaCl}$. The expression of all eight genes increased rapidly in the roots and reached a maximum level 12 hours after the start of treatment. In shoots, five of the genes had highest expression levels at 12 hours, and two genes had highest expression levels at 6 hours. AcoDREB06 expression in shoots decreased after salt treatment. The differential responses of the AcoDREB genes after $\mathrm{NaCl}$ treatment suggest that they have distinct roles in salt stress response (Fig. 9 A-H).

To analyze the response to drought stress, we treated plants with $350 \mathrm{mM}$ mannitol. In the shoots, six genes (AcoDREB01, 03, 11, 14, 18, and 19) were down-regulated after 12 hours.

$A c o D R E B 09$ was extremely sensitive to drought stress, and its expression level quickly reached a maximum at 6 hours after treatment. Except for AcoDREB06, the expression levels of the analyzed genes did not change as much in the roots as they did in the shoots. Compared to the control plants, $A c o D R E B 03$ and $A c o D R E B 11$ were rapidly down-regulated in the roots. These expression pattern changes after mannitol treatment indicate the vital role played by $A c o D R E B$ genes in response to drought conditions (Fig. 9 I-P).

Cold stress drastically affects plant growth and development and causes major crop yield losses (Cai et al. 2015). The expression levels of the DREB genes were equally affected by cold treatment in the roots and in the shoots. In particular, three genes (AcoDREB01, 03, and 18) responded rapidly to cold treatment, and their expression levels in the shoots peaked at 6 hours. Two genes (AcoDREB09 and AcoDREB19) reached their maximum expression levels in the shoots after 48 hours (Fig. 9 Q-X). 
355 To analyze the effects of heat stress, the plants were subjected to $45^{\circ} \mathrm{C}$ temperature. In the 356 shoots, the majority of the analyzed genes were initially down-regulated then subsequently up-

357 regulated. $A c o D R E B 03$ was the only gene that was up-regulated in the shoots during the first 12 358 hours. In the roots, the expression levels of four genes (AcoDREB01, 09, 11, and 18) gradually 359 increased and peaked at 48 hours. The expression levels of two genes (AcoDREB03 and 360 AcoDREB 14) decreased rapidly after exposure to high temperature stress. Unlike the other genes, 361 the expression of $A c o D R E B 06$ in the roots peaked at 12 hours. Collectively, these results indicate 362 the involvement of $A c o D R E B$ genes in the response to heat stress in pineapple (Fig. 9 Y-FF).

363

\section{DISCUSSION}

365 Climate change has drawn attention to the detrimental effects of environmental stress on plant growth and yield (Chinnusamy et al. 2004; Mittler 2006; Suzuki et al. 2014). Throughout their development, plants respond to stress by activating genes that induce a specific response to the stressor. These genes can be roughly divided into two categories. The first group includes functional genes directly responsible for the production of important stress resistance proteins, such as aquaporin, LEA protein, and antioxidant enzymes. The second group includes genes that encode regulatory proteins, such as TFs and protein kinases.

372

373

By recognizing and binding specific promoter cis-elements, TFs regulate the transcription of downstream genes. There are hundreds of TFs in higher plants, and they have important roles in plant reproductive development and physiological metabolism (Liu et al. 2001). In response to environmental stress, TFs regulate plant growth and development by controlling a variety of downstream genes. The AtMYB4 TF protects plants from the harmful effects of UV radiation (Hemm et al. 2001). Transgenic expression of GmMYB22 in Arabidopsis enhances drought tolerance, salt tolerance, and ABA sensitivity (Shan et al. 2012). One class of bZIP proteins, the TGA/OBF family members, interact with NPR1 in the salicylic acid (SA) defense signaling pathway (Singh et al. 2002).

The DREB TFs contain a conserved AP2/EREBP domain, which is involved in the response to environmental stress. DREBs regulate genes that enhance plant stress tolerance by interacting with DRE cis-elements. In experiments with mutated DRE binding sites, DREB TF binding was abolished (Dubouzet et al. 2003; Liu et al. 1998). Other experiments dissected the preferential binding of DREB1A to two DRE sequences in Arabidopsis and Oryza sativa (Dubouzet et al. 2003; Sakuma et al. 2002).

Several studies have elucidated the functions and evolutionary history of $D R E B$ genes in many plant species including Arabidopsis, rice, and maize. There have also been a growing number of studies that report the functions of $D R E B$ genes in stress response. $D R E B$ genes were first cloned in Arabidopsis in 1998 (Liu et al. 1998). DREB1 and DREB2 were involved in two separate signal transduction pathways that protect plants from low-temperature and dehydration 
395

396

397

398

399

400

401

402

403

404

405

406

407

408

409

410

411

412

413

414

415

416

417

418

419

420

421

422

423

424

425

426

427

428

429

430

431

432

433

434

conditions (Liu et al. 1998). In Arabidopsis, the expression of VuDREB2a from the legume cowpea (Vigna unguiculata) was found to enhance drought resistance (Sadhukhan et al. 2014). DREBs also protect plants from biotic and abiotic stress by regulating anthocyanin biosynthesis (Song et al. 2019). In addition, MaDREB1-MaDREB4 (Achr9G04630, Achr5G280, Achr6G32780, and Achr11G24820) are induced by ethylene in bananas (Musa acuminata) and regulate fruit ripening (Kuang et al. 2017). These examples from diverse plant species indicate that DREBs contribute significantly to plant growth and development.

Considering its high economic value, pineapple production would benefit tremendously from an improved understanding of the stress tolerance mechanisms in this species. We identified pineapple $D R E B$ genes and gathered the following information: the predicted isoelectric points $(p I)$ and molecular weights of the encoded proteins, chromosome location, gene structure and motif, phylogenetic relationships, domain architecture, promoter cis-elements, and expression profiles under abiotic stress.

We identified 20 AcoDREB genes (Table 1), which is fewer than the number of DREB genes in other monocots. For example, there are 57 OsDREBs (Rashid et al. 2012; Nakano et al. 2006) (Oryza sativa), 51 ZmDREBs (Du et al. 2014) (Zea mays), 52 SbDREBs (Yan et al. 2013) (Sorghum bicolor), and 27 PeDREBs (Wu et al. 2015) (Phyllostachys edulis). The lower number in pineapple suggests that some genes may have been lost during the evolution of this species. The predicted AcoDREB proteins ranged from 149 (AcoDREB13) to 463 (AcoDREB20) amino acids. The average length was 255 amino acids, which is very similar to that in rice and Chinese jujube (Ziziphus jujube Mill) (Zhang \& Li 2018). The predicted molecular weights (Mw) ranged from 16.32 (AcoDREB13) to 49.3 (AcoDREB20) $\mathrm{kDa}$, and the predicted $\mathrm{pI}$ values ranged from 4.71 (AcoDREB 10) to 9.68 (AcoDREB07) (Table 1). The ranges reported in other species include the following: $12.13-59.27 \mathrm{kDa}$ and 4.6-10.64 pI in pepper (Capsicum annuum L.) (Jin et al. 2018 ) and 17.6-36.3 kDa and 4.5-11.07 pI in moso bamboo (Phyllostachys edulis) (Wu et al. 2015). The predicted $\mathrm{Mw}$ and $\mathrm{pI}$ ranges in pineapple are roughly similar to those reported in other species, indicating some degree of conservation in the biochemistry and function of DREB TFs in plants. Therefore, based on previous studies of DREBs in other species, we can propose and test hypotheses about the characteristics and functions of DREBs.

To investigate the phylogenetic relationships of the AcoDREB gene family, we constructed an unrooted phylogenetic tree based on multiple sequence alignment of DREB amino acid sequences from pineapple, Arabidopsis, and rice. The comparative analysis classified the $A c o D R E B$ genes into five subgroups (Fig. 3), and the numbers of genes in subgroups I to $\mathrm{V}$ were 3, 4, 4, 5, and 4, respectively (Fig. 3). In Arabidopsis, the DREB genes can be divided into six subgroups (A1-A6), with only one gene in the A3 subgroup. In the current study, AcoDREB04, $A T 2 G 40220$ (A3 subgroup), and $A T 3 G 57600$ (A2 subgroup) were on the same branch of the phylogenetic tree (Fig. 3), but we ultimately grouped AcoDREB04 with the A2 subgroup based 
435 on sequence and domain analysis (Nakano et al. 2006). As a result, there were no AcoDREB 436 genes that grouped together with the A3 subgroup. The genes of A3 subgroup may have been 437 lost during the evolution of these species.

438

439

440

441

442

443

444

445

446

447

448

449

450

451

452

453

454

455

456

457

458

459

460

461

462

463

464

465

466

467

468

469

470

471

472

473

Analysis of the intron-exon structure of $A c o D R E B$ genes revealed a small number of introns. AcoDREB05 had the highest number of introns (three), while many of the other genes lacked introns, which is consistent with previous reports in grape (Vitis vinifera) and jujube (Zhao et al. 2014; Zhang \& Li 2018). Some studies have proposed that introns could delay regulatory responses. To respond quickly to various stresses, genes must be rapidly activated. Having fewer introns would assist this process (Jeffares et al. 2008). In support of this hypothesis, we found a quick response to salt stress in the eight genes that we examined (Fig. 9).

The expression patterns of some $A c o D R E B$ genes resembled the expression patterns of homologs in other species. AcoDREB19 was highly expressed in anthers (Fig. 7, Fig. 8), which is similar to the expression of its homolog in rice (LOC_Os08g27220) (Davidson et al. 2012). Similarly, AcoDREB16 and its homolog in rice (LOC_Os 10g22600) are both highly expressed in roots. OSDREB2A, when overexpressed in rice, enhances salt stress tolerance (Mallikarjuna et al. 2011), without changing its total nutritional composition (Cornwell 2014; Cho et al. 2016). Our analysis suggested that overexpression of some AcoDREBs in pineapple could help to develop new pineapple varieties with abiotic stress tolerance. Furthermore, we found that $A c o D R E B 05$, 16, and 17 displayed high expression levels in fruits (Fig. 7), indicating that they may play an important role in fruit development. Therefore, it is possible that these genes may have applications in improving fruit quality through molecular breeding.

$D R E B$ genes respond to stress conditions through differential expression in shoots and roots (Torres et al. 2019). We therefore quantified the transcript levels of eight AcoDREB genes in pineapple seedlings subjected to different abiotic stress conditions. Under salt stress, eight of the DREB genes displayed similar expression patterns, and were induced in both shoots and roots (Fig. 9 A-H). Previous studies have reported that A1 subgroup members play important roles in the response to salt and drought stress in Arabidopsis (Yamaguchi-Shinozaki \& Shinozaki 2006). In our study, AcoDREB01 and AcoDREB03 from subgroup I were induced in plants subjected to salt and drought stress (Fig. 9 A-B, I-J). Specifically, they were expressed in roots under salt stress, and in shoots under drought stress. These two genes also had similar expression patterns over the course of treatment, indicating that they may be coordinately regulated in response to salt and drought stress. Previous studies showed that ScDREB10 was up-regulated after $\mathrm{NaCl}$ $(150 \mathrm{mM})$ treatment and that its overexpression enhanced salt stress tolerance in Arabidopsis seedlings ( $L i$ et al. 2019; Li et al. 2016). We therefore infer that AcoDREB01 and AcoDREB03 may perform similar functions in pineapple. 
474 Subgroup IV members AcoDREB11 and AcoDREB14 were both up-regulated under salt 475 treatment and cold stress (Fig. 9). These expression changes are similar to those of the A5 476 subgroup member GmDREB2 (Chen et al. 2007), suggesting functional conservation of these 477 homologs in pineapple and soybean. At the same time, they also indicate functional conservation 478 of the genes that belong to the same subgroup. Under various abiotic stresses, AcoDREB06 479 expression decreased in the leaves and increased in the roots, indicating that enhanced expression 480 of this gene could improve the resistance of roots to different abiotic stresses. On the other hand, 481 the decreased expression of AcoDREB06 in shoots suggests that it may also regulate other 482 pathways that are critical to plant survival (Fig. 9). For instance, similar to the Arabidopsis gene $483 \operatorname{HARDY}(A T 2 G 36450)$, it may improve drought and salt tolerance by reducing transpiration

484

485

486

487

488

489

490

491

492

493

494

495

496

497

498

499

500

501

502

503

504

505

506

507

508

509

510

511

512

513 (Abogadallah et al. 2011). The RNA-Seq data indicated that AcoDREB19 had very low expression in roots, but its expression increased significantly under different abiotic stresses.

The expression analysis for the eight selected genes were mostly in line with our expectations based on the predicted cis-elements in their promoters (Fig. 4, Fig. 9). TC-rich and W-box elements were found in the promoters of AcoDREB01, 06, 09, 11, and 19. Since these ciselements have been identified upstream to genes that are key to plant defense in other species (Laloi et al. 2004; Xu et al. 2010), we speculate that these four genes play a similar role in resistance to pineapple diseases (Hubert et al. 2014; Calderon-Arguedas et al. 2015). These genes could potentially be used to breed disease-resistant pineapple seedlings.

\section{CONCLUSIONS}

We identified 20 AcoDREB genes in pineapple, and collected information about their gene structures and expression profiles under various abiotic stresses. To the best of our knowledge, this is the first genome-wide analysis of $D R E B$ genes in pineapple. We have shown that $A c o D R E B$ genes respond to a variety of abiotic stresses (drought, high salt, high- and lowtemperature stress). Our findings provide preliminary data for further functional analysis of $A c o D R E B$ genes in pineapple, and information for developing new pineapple varieties with important agronomic traits such as stress tolerance.

\section{ACKNOWLEDGEMENTS}

We would like to thank the reviewers for their helpful comments on the original manuscript. This work was supported by NSFC (U1605212, 31761130074, 31970333), a Guangxi Distinguished Experts Fellowship, and a Newton Advanced Fellowship (NA160391).

\section{REFERENCES}

Abogadallah GM, Nada RM, Malinowski R, and Quick P. 2011. Overexpression of HARDY, an AP2/ERF gene from Arabidopsis, improves drought and salt tolerance by reducing transpiration and sodium uptake in transgenic Trifolium alexandrinum L. Planta 233:12651276. $10.1007 / \mathrm{s} 00425-011-1382-3$ 
514

515

516

517

518

519

520

521

522

523

524

525

526

527

528

529

530

531

532

533

534

535

536

537

538

539

540

541

542

543

544

545

546

547

548

549

550

551

552

553

554

555

556

557

558

559

Agarwal PK, Agarwal P, Reddy MK, and Sopory SK. 2006. Role of DREB transcription factors in abiotic and biotic stress tolerance in plants. Plant Cell Rep 25:1263-1274. 10.1007/s00299-006-0204-8

Agarwal PK, Gupta K, Lopato S, and Agarwal P. 2017. Dehydration responsive element binding transcription factors and their applications for the engineering of stress tolerance. $J$ Exp Bot 68:2135-2148. 10.1093/jxb/erx118

Augustine SM, Narayan JA, Syamaladevi DP, Appunu C, Chakravarthi M, Ravichandran V, Tuteja N, and Subramonian N. 2015. Overexpression of EaDREB2 and pyramiding of EaDREB2 with the pea DNA helicase gene (PDH45) enhance drought and salinity tolerance in sugarcane (Saccharum spp. hybrid). Plant Cell Reports 34:247-263. 10.1007/s00299-0141704-6

Azam SM, Liu Y, Rahman ZU, Ali H, Yan C, Wang L, Priyadarshani SVGN, Hu B, Huang $\mathbf{X}$, Xiong J, and Qin Y. 2018. Identification, Characterization and Expression Profiles of Dof Transcription Factors in Pineapple (Ananas comosus L). Tropical Plant Biology 11:49-64. 10.1007/s12042-018-9200-8

Bailey TL, Boden M, Buske FA, Frith M, Grant CE, Clementi L, Ren J, Li WW, and Noble WS. 2009. MEME SUITE: tools for motif discovery and searching. Nucleic Acids Res 37:W202-208. 10.1093/nar/gkp335

Berardini TZ, Reiser L, Li D, Mezheritsky Y, Muller R, Strait E, and Huala E. 2015. The Arabidopsis information resource: Making and mining the "gold standard" annotated reference plant genome. Genesis 53:474-485. 10.1002/dvg.22877

Cai H, Cheng J, Yan Y, Xiao Z, Li J, Mou S, Qiu A, Lai Y, Guan D, and He S. 2015. Genome-wide identification and expression analysis of calcium-dependent protein kinase and its closely related kinase genes in Capsicum annuum. Front Plant Sci 6:737. 10.3389/fpls.2015.00737

Cai H, Zhang M, Liu Y, He Q, Chai M, Liu L, Chen F, Huang Y, Yan M, and Zhao H. 2019. Genome-Wide Classification and Evolutionary and Functional Analyses of the VQ Family. Tropical Plant Biology:1-15.

Cai H, Zhao L, Wang L, Zhang M, Su Z, Cheng Y, Zhao H, and Qin Y. 2017. ERECTA signaling controls Arabidopsis inflorescence architecture through chromatin-mediated activation of PRE1 expression. New Phytol 214:1579-1596. 10.1111/nph.14521

Calderon-Arguedas O, Troyo A, Moreira-Soto RD, Marin R, and Taylor L. 2015. Dengue viruses in Aedes albopictus Skuse from a pineapple plantation in Costa Rica. J Vector Ecol 40:184-186. 10.1111/jvec.12149

Century K, Reuber TL, and Ratcliffe OJ. 2008. Regulating the regulators: the future prospects for transcription-factor-based agricultural biotechnology products. Plant Physiol 147:20-29. 10.1104/pp.108.117887

Chen M, Wang QY, Cheng XG, Xu ZS, Li LC, Ye XG, Xia LQ, and Ma YZ. 2007. GmDREB2, a soybean DRE-binding transcription factor, conferred drought and high-salt tolerance in transgenic plants. Biochem Biophys Res Commun 353:299-305. 10.1016/j.bbrc.2006.12.027

Chen P, Li Y, Zhao L, Hou Z, Yan M, Hu B, Liu Y, Azam SM, Zhang Z, Rahman ZU, Liu L, and Qin Y. 2017. Genome-Wide Identification and Expression Profiling of ATP-Binding Cassette (ABC) Transporter Gene Family in Pineapple (Ananas comosus (L.) Merr.) Reveal the Role of AcABCG38 in Pollen Development. Front Plant Sci 8:2150.

10.3389/fpls.2017.02150

Peer) reviewing PDF | (2019:09:41145:5:0:NEW 14 Mar 2020) 
560 Chernomor O, von Haeseler A, and Minh BQ. 2016. Terrace Aware Data Structure for 
605

606

607

608

609

610

611

612

613

614

615

616

617

618

619

620

621

622

623

624

625

626

627

628

629

630

631

632

633

634

635

636

637

638

639

640

641

642

643

644

645

646

647

648

649

650

Golldack D, Luking I, and Yang O. 2011. Plant tolerance to drought and salinity: stress regulating transcription factors and their functional significance in the cellular transcriptional network. Plant Cell Rep 30:1383-1391. 10.1007/s00299-011-1068-0

Gumi AM, Guha PK, Mazumder A, Jayaswal P, and Mondal TK. 2018. Characterization of OglDREB2A gene from African rice (Oryza glaberrima), comparative analysis and its transcriptional regulation under salinity stress. 3 Biotech 8:91. 10.1007/s13205-018-1098-1

Guo AY, Zhu QH, Chen X, and Luo JC. 2007. [GSDS: a gene structure display server]. $Y i$ Chuan 29:1023-1026.

Hemm MR, Herrmann KM, and Chapple C. 2001. AtMYB4: a transcription factor general in the battle against UV. Trends Plant Sci 6:135-136.

Hoang DT, Chernomor O, von Haeseler A, Minh BQ, and Vinh LS. 2018. UFBoot2: Improving the Ultrafast Bootstrap Approximation. Mol Biol Evol 35:518-522. 10.1093/molbev/msx281

Hubert J, Fourrier C, Laplace D, and Ioos R. 2014. First Report of Pineapple Black Rot Caused by Ceratocystis paradoxa on Ananas comosus in French Guiana. Plant Dis 98:1584. 10.1094/PDIS-05-14-0510-PDN

Hwang JE, Lim CJ, Chen H, Je J, Song C, and Lim CO. 2012. Overexpression of Arabidopsis dehydration- responsive element-binding protein $2 \mathrm{C}$ confers tolerance to oxidative stress. Mol Cells 33:135-140. 10.1007/s10059-012-2188-2

Jaglo-Ottosen KR, Gilmour SJ, Zarka DG, Schabenberger O, and Thomashow MF. 1998. Arabidopsis CBF1 overexpression induces COR genes and enhances freezing tolerance. Science 280:104-106. 10.1126/science.280.5360.104

Jeffares DC, Penkett CJ, and Bahler J. 2008. Rapidly regulated genes are intron poor. Trends Genet 24:375-378. 10.1016/j.tig.2008.05.006

Jiang J, Ma S, Ye N, Jiang M, Cao J, and Zhang J. 2017. WRKY transcription factors in plant responses to stresses. J Integr Plant Biol 59:86-101. 10.1111/jipb.12513

Jin JH, Wang M, Zhang HX, Khan A, Wei AM, Luo DX, and Gong ZH. 2018. Genomewide identification of the AP2/ERF transcription factor family in pepper (Capsicum annuum L.). Genome 61:663-674. 10.1139/gen-2018-0036

Kawahara Y, de la Bastide M, Hamilton JP, Kanamori H, McCombie WR, Ouyang S, Schwartz DC, Tanaka T, Wu JZ, Zhou SG, Childs KL, Davidson RM, Lin HN, Quesada-Ocampo L, Vaillancourt B, Sakai H, Lee SS, Kim J, Numa H, Itoh T, Buell CR, and Matsumoto T. 2013. Improvement of the Oryza sativa Nipponbare reference genome using next generation sequence and optical map data. Rice 6. Artn 4

$10.1186 / 1939-8433-6-4$

Kim YH, Yang KS, Ryu SH, Kim KY, Song WK, Kwon SY, Lee HS, Bang JW, and Kwak SS. 2008. Molecular characterization of a cDNA encoding DRE-binding transcription factor from dehydration-treated fibrous roots of sweetpotato. Plant Physiol Biochem 46:196-204. 10.1016/j.plaphy.2007.09.012

Kuang JF, Chen JY, Liu XC, Han YC, Xiao YY, Shan W, Tang Y, Wu KQ, He JX, and Lu WJ. 2017. The transcriptional regulatory network mediated by banana (Musa acuminata) dehydration-responsive element binding (MaDREB) transcription factors in fruit ripening. New Phytol 214:762-781. 10.1111/nph.14389

Kumar S, Stecher G, and Tamura K. 2016. MEGA7: Molecular Evolutionary Genetics Analysis Version 7.0 for Bigger Datasets. Mol Biol Evol 33:1870-1874. $10.1093 / \mathrm{molbev} / \mathrm{msw} 054$

Peer) reviewing PDF | (2019:09:41145:5:0:NEW 14 Mar 2020) 
651

652

653

654

655

656

657

658

659

660

661

662

663

664

665

666

667

668

669

670

671

672

673

674

675

676

677

678

679

680

681

682

683

684

685

686

687

688

689

690

691

692

693

694

695

696
Laloi C, Mestres-Ortega D, Marco Y, Meyer Y, and Reichheld JP. 2004. The Arabidopsis cytosolic thioredoxin h5 gene induction by oxidative stress and its W-box-mediated response to pathogen elicitor. Plant Physiol 134:1006-1016. 10.1104/pp.103.035782

Lescot M, Dehais P, Thijs G, Marchal K, Moreau Y, Van de Peer Y, Rouze P, and Rombauts S. 2002. PlantCARE, a database of plant cis-acting regulatory elements and a portal to tools for in silico analysis of promoter sequences. Nucleic Acids Res 30:325-327. 10.1093/nar/30.1.325

Letunic I, and Bork P. 2018. 20 years of the SMART protein domain annotation resource. Nucleic Acids Research 46:D493-D496. 10.1093/nar/gkx922

Li H, Zhang D, Li X, Guan K, and Yang H. 2016. Novel DREB A-5 subgroup transcription factors from desert moss (Syntrichia caninervis) confers multiple abiotic stress tolerance to yeast. J Plant Physiol 194:45-53. 10.1016/j.jplph.2016.02.015

Li S, Zhao Q, Zhu D, and Yu J. 2018. A DREB-Like Transcription Factor From Maize (Zea mays), ZmDREB4.1, Plays a Negative Role in Plant Growth and Development. Front Plant Sci 9:395. 10.3389/fpls.2018.00395

Li X, Liang Y, Gao B, Mijiti M, Bozorov TA, Yang H, Zhang D, and Wood AJ. 2019. ScDREB10, an A-5c type of DREB Gene of the Desert Moss Syntrichia caninervis, Confers Osmotic and Salt Tolerances to Arabidopsis. Genes (Basel) 10. 10.3390/genes10020146

Liang Y, Li X, Zhang D, Gao B, Yang H, Wang Y, Guan K, and Wood AJ. 2017. ScDREB8, a novel A-5 type of DREB gene in the desert moss Syntrichia caninervis, confers salt tolerance to Arabidopsis. Plant Physiol Biochem 120:242-251. 10.1016/j.plaphy.2017.09.014

Liu Q, Kasuga M, Sakuma Y, Abe H, Miura S, Yamaguchi-Shinozaki K, and Shinozaki K. 1998. Two transcription factors, DREB1 and DREB2, with an EREBP/AP2 DNA binding domain separate two cellular signal transduction pathways in drought- and low-temperatureresponsive gene expression, respectively, in Arabidopsis. Plant Cell 10:1391-1406. $10.1105 /$ tpc.10.8.1391

Liu Q, Zhang G, and Chen S. 2001. Structure and regulatory function of plant transcription factors. Chinese Science Bulletin 46:271-278. 10.1007/bf03187184

Lobo G, and Paull R. 2016. Handbook of Pineapple Technology: Postharvest Science, Processing and Nutrition.

Malhotra S, and Sowdhamini R. 2014. Interactions Among Plant Transcription Factors Regulating Expression of Stress-responsive Genes. Bioinform Biol Insights 8:193-198. 10.4137/BBI.S16313

Mallikarjuna G, Mallikarjuna K, Reddy MK, and Kaul T. 2011. Expression of OsDREB2A transcription factor confers enhanced dehydration and salt stress tolerance in rice (Oryza sativa L.). Biotechnol Lett 33:1689-1697. 10.1007/s10529-011-0620-x

Matsukura S, Mizoi J, Yoshida T, Todaka D, Ito Y, Maruyama K, Shinozaki K, and Yamaguchi-Shinozaki K. 2010. Comprehensive analysis of rice DREB2-type genes that encode transcription factors involved in the expression of abiotic stress-responsive genes. $\mathrm{Mol}$ Genet Genomics 283:185-196. 10.1007/s00438-009-0506-y

Ming R, VanBuren R, Wai CM, Tang H, Schatz MC, Bowers JE, Lyons E, Wang ML, Chen J, Biggers E, Zhang J, Huang L, Zhang L, Miao W, Zhang J, Ye Z, Miao C, Lin Z, Wang H, Zhou H, Yim WC, Priest HD, Zheng C, Woodhouse M, Edger PP, Guyot R, Guo HB, Guo H, Zheng G, Singh R, Sharma A, Min X, Zheng Y, Lee H, Gurtowski J, Sedlazeck FJ, Harkess A, McKain MR, Liao Z, Fang J, Liu J, Zhang X, Zhang Q, Hu W, Qin Y, Wang K, Chen LY, Shirley N, Lin YR, Liu LY, Hernandez AG, Wright CL,

Peer) reviewing PDF | (2019:09:41145:5:0:NEW 14 Mar 2020) 
700

701

702

703

704

705

706

707

708

709

710

711

712

713

714

715

716

717

718

719

720

721

722

723

724

725

726

727

728

729

730

731

732

733

734

735

736

737

738

739

740

741

742

Bulone V, Tuskan GA, Heath K, Zee F, Moore PH, Sunkar R, Leebens-Mack JH, Mockler T, Bennetzen JL, Freeling M, Sankoff D, Paterson AH, Zhu X, Yang X, Smith JA, Cushman JC, Paull RE, and Yu Q. 2015. The pineapple genome and the evolution of CAM photosynthesis. Nat Genet 47:1435-1442. 10.1038/ng.3435

Mittler R. 2006. Abiotic stress, the field environment and stress combination. Trends Plant Sci 11:15-19. 10.1016/j.tplants.2005.11.002

Mondini L, Nachit MM, and Pagnotta MA. 2015. Allelic variants in durum wheat (Triticum turgidum L. var. durum) DREB genes conferring tolerance to abiotic stresses. Mol Genet Genomics 290:531-544. 10.1007/s00438-014-0933-2

Moyle R, Fairbairn DJ, Ripi J, Crowe M, and Botella JR. 2005. Developing pineapple fruit has a small transcriptome dominated by metallothionein. Journal of Experimental Botany 56:101-112. DOI 10.1093/jxb/eri015

Nakano T, Suzuki K, Fujimura T, and Shinshi H. 2006. Genome-wide analysis of the ERF gene family in Arabidopsis and rice. Plant Physiol 140:411-432. 10.1104/pp. 105.073783

Narusaka Y, Nakashima K, Shinwari ZK, Sakuma Y, Furihata T, Abe H, Narusaka M, Shinozaki K, and Yamaguchi-Shinozaki K. 2003. Interaction between two cis-acting elements, ABRE and DRE, in ABA-dependent expression of Arabidopsis rd29A gene in response to dehydration and high-salinity stresses. Plant J 34:137-148. 10.1046/j.1365313x.2003.01708.x

Nguyen LT, Schmidt HA, von Haeseler A, and Minh BQ. 2015. IQ-TREE: a fast and effective stochastic algorithm for estimating maximum-likelihood phylogenies. Mol Biol Evol 32:268-274. 10.1093/molbev/msu300

Okamuro JK, Caster B, Villarroel R, Van Montagu M, and Jofuku KD. 1997. The AP2 domain of APETALA2 defines a large new family of DNA binding proteins in Arabidopsis. Proc Natl Acad Sci U S A 94:7076-7081. 10.1073/pnas.94.13.7076

Priyadarshani S, Cai H, Zhou Q, Liu Y, Cheng Y, Xiong J, Patson DL, Cao S, Zhao H, and Qin Y. 2019. An Efficient Agrobacterium Mediated Transformation of Pineapple with GFPTagged Protein Allows Easy, Non-Destructive Screening of Transgenic Pineapple Plants. Biomolecules 9. 10.3390/biom9100617

Priyadarshani S, Hu B, Li W, Ali H, Jia H, Zhao L, Ojolo SP, Azam SM, Xiong J, Yan M, Ur Rahman Z, Wu Q, and Qin Y. 2018. Simple protoplast isolation system for gene expression and protein interaction studies in pineapple (Ananas comosus L.). Plant Methods 14:95. 10.1186/s13007-018-0365-9

Qin F, Kakimoto M, Sakuma Y, Maruyama K, Osakabe Y, Tran LS, Shinozaki K, and Yamaguchi-Shinozaki K. 2007. Regulation and functional analysis of ZmDREB2A in response to drought and heat stresses in Zea mays L. Plant J 50:54-69. 10.1111/j.1365313X.2007.03034.X

Rahman Zu, Azam SM, Liu Y, Yan C, Ali H, Zhao L, Chen P, Yi L, Priyadarshani SVGN, and Yuan Q. 2017. Expression Profiles of Wuschel-Related Homeobox Gene Family in Pineapple (Ananas comosus L). Tropical Plant Biology 10:204-215. 10.1007/s12042-0179192-9

Rashid M, Guangyuan H, Guangxiao Y, Hussain J, and Xu Y. 2012. AP2/ERF Transcription Factor in Rice: Genome-Wide Canvas and Syntenic Relationships between Monocots and Eudicots. Evol Bioinform Online 8:321-355. 10.4137/EBO.S9369

Ray DK, Mueller ND, West PC, and Foley JA. 2013. Yield Trends Are Insufficient to Double Global Crop Production by 2050. PLoS One 8:e66428. 10.1371/journal.pone.0066428

Peer) reviewing PDF | (2019:09:41145:5:0:NEW 14 Mar 2020) 
Roy Choudhury S, Roy S, Das R, and Sengupta DN. 2008. Differential transcriptional

744

745

746

747

748

749

750

751

752

753

754

755

756

757

758

759

760

761

762

763

764

765

766

767

768

769

770

771

772

773

774

775

776

777

778

779

780

781

782

783

784

785

786 wounding, low temperature and different photoperiods during fruit ripening and functional analysis of banana SPS gene promoter. Planta 229:207-223. 10.1007/s00425-008-0821-2

Sadhukhan A, Kobayashi Y, Kobayashi Y, Tokizawa M, Yamamoto YY, Iuchi S, Koyama H, Panda SK, and Sahoo L. 2014. VuDREB2A, a novel DREB2-type transcription factor in the drought-tolerant legume cowpea, mediates DRE-dependent expression of stressresponsive genes and confers enhanced drought resistance in transgenic Arabidopsis. Planta 240:645-664. 10.1007/s00425-014-2111-5

Sakuma Y, Liu Q, Dubouzet JG, Abe H, Shinozaki K, and Yamaguchi-Shinozaki K. 2002. DNA-binding specificity of the ERF/AP2 domain of Arabidopsis DREBs, transcription factors involved in dehydration- and cold-inducible gene expression. Biochem Biophys Res Commun 290:998-1009. 10.1006/bbrc.2001.6299

Sazegari S, Niazi A, and Ahmadi FS. 2015. A study on the regulatory network with promoter analysis for Arabidopsis DREB-genes. Bioinformation 11:101-106. 10.6026/97320630011101

Shan H, Chen S, Jiang J, Chen F, Chen Y, Gu C, Li P, Song A, Zhu X, Gao H, Zhou G, Li T, and Yang X. 2012. Heterologous expression of the chrysanthemum R2R3-MYB transcription factor CmMYB2 enhances drought and salinity tolerance, increases hypersensitivity to ABA and delays flowering in Arabidopsis thaliana. Mol Biotechnol 51:160-173. 10.1007/s12033-011-9451-1

Singh K, Foley RC, and Onate-Sanchez L. 2002. Transcription factors in plant defense and stress responses. Curr Opin Plant Biol 5:430-436.

Song T, Li K, Wu T, Wang Y, Zhang X, Xu X, Yao Y, and Han Z. 2019. Identification of new regulators through transcriptome analysis that regulate anthocyanin biosynthesis in apple leaves at low temperatures. PLoS One 14:e0210672. 10.1371/journal.pone.0210672

Su Z, Wang L, Li W, Zhao L, Huang X, Azam SM, and Qin Y. 2017. Genome-Wide Identification of Auxin Response Factor (ARF) Genes Family and its Tissue-Specific Prominent Expression in Pineapple (Ananas comosus). Tropical Plant Biology 10:86-96. 10.1007/s12042-017-9187-6

Suzuki N, Rivero RM, Shulaev V, Blumwald E, and Mittler R. 2014. Abiotic and biotic stress combinations. New Phytol 203:32-43. 10.1111/nph.12797

Torres LF, Reichel T, Déchamp E, Aquino SOd, Duarte KE, Alves GSC, Silva AT, Cotta MG, Costa TS, Diniz LEC, Breitler J-C, Collin M, Paiva LV, Andrade AC, Etienne H, and Marraccini P. 2019. Expression of DREB-Like Genes in Coffea canephora and C. arabica Subjected to Various Types of Abiotic Stress. Tropical Plant Biology:1-19. 10.1007/s12042-019-09223-5

Trapnell C, Roberts A, Goff L, Pertea G, Kim D, Kelley DR, Pimentel H, Salzberg SL, Rinn JL, and Pachter L. 2012. Differential gene and transcript expression analysis of RNAseq experiments with TopHat and Cufflinks. Nat Protoc 7:562-578. 10.1038/nprot.2012.016

Urao T, Yamaguchi-Shinozaki K, Urao S, and Shinozaki K. 1993. An Arabidopsis myb homolog is induced by dehydration stress and its gene product binds to the conserved MYB recognition sequence. Plant Cell 5:1529-1539. 10.1105/tpc.5.11.1529

Voorrips RE. 2002. MapChart: software for the graphical presentation of linkage maps and QTLs. J Hered 93:77-78. 10.1093/jhered/93.1.77 
787

788

789

790

791

792

793

794

795

796

797

798

799

800

801

802

803

804

805

806

807

808

809

810

811

812

813

814

815

816

817

818

819

820

821

Wang WQ. 2016. The Molecular Detection of Corynespora Cassiicola on Cucumber by PCR Assay Using DNAman Software and NCBI. Computer and Computing Technologies in Agriculture Ix, Ccta 2015, Pt Ii 479:248-258. 10.1007/978-3-319-48354-2_26

Wu H, Lv H, Li L, Liu J, Mu S, Li X, and Gao J. 2015. Genome-Wide Analysis of the AP2/ERF Transcription Factors Family and the Expression Patterns of DREB Genes in Moso Bamboo (Phyllostachys edulis). PLoS One 10:e0126657. 10.1371/journal.pone.0126657

Xiong Y, and Fei SZ. 2006. Functional and phylogenetic analysis of a DREB/CBF-like gene in perennial ryegrass (Lolium perenne L.). Planta 224:878-888. 10.1007/s00425-006-0273-5

Xu H, Yu Q, Shi Y, Hua X, Tang H, Yang L, Ming R, and Zhang J. 2018. PGD: Pineapple Genomics Database. Hortic Res 5:66. 10.1038/s41438-018-0078-2

Xu W, Yu Y, Ding J, Hua Z, and Wang Y. 2010. Characterization of a novel stilbene synthase promoter involved in pathogen- and stress-inducible expression from Chinese wild Vitis pseudoreticulata. Planta 231:475-487. 10.1007/s00425-009-1062-8

Yamaguchi-Shinozaki K, and Shinozaki K. 1993. Arabidopsis DNA encoding two desiccation-responsive rd29 genes. Plant Physiol 101:1119-1120. 10.1104/pp.101.3.1119

Yamaguchi-Shinozaki K, and Shinozaki K. 2006. Transcriptional regulatory networks in cellular responses and tolerance to dehydration and cold stresses. Annu Rev Plant Biol 57:781803. 10.1146/annurev.arplant.57.032905.105444

Yan HW, Hong L, Zhou YQ, Jiang HY, Zhu SW, Fan J, and Cheng BJ. 2013. A genomewide analysis of the ERF gene family in sorghum. Genet Mol Res 12:2038-2055. 10.4238/2013.May.13.1

Yang Y, Wu J, Zhu K, Liu L, Chen F, and Yu D. 2009. Identification and characterization of two chrysanthemum (Dendronthema x moriforlium) DREB genes, belonging to the AP2/EREBP family. Mol Biol Rep 36:71-81. 10.1007/s11033-007-9153-8

Zhang M, Liu Y, Shi H, Guo M, Chai M, He Q, Yan M, Cao D, Zhao L, Cai H, and Qin Y. 2018. Evolutionary and expression analyses of soybean basic Leucine zipper transcription factor family. BMC Genomics 19:159. 10.1186/s12864-018-4511-6

Zhang Z, and Li XG. 2018. Genome-wide identification of AP2/ERF superfamily genes and their expression during fruit ripening of Chinese jujube. Scientific Reports 8. ARTN 15612 10.1038/s41598-018-33744-w

Zhao T, Xia H, Liu J, and Ma F. 2014. The gene family of dehydration responsive elementbinding transcription factors in grape (Vitis vinifera): genome-wide identification and analysis, expression profiles, and involvement in abiotic stress resistance. Mol Biol Rep 41:1577-1590. 10.1007/s11033-013-3004-6

PeerJ reviewing PDF | (2019:09:41145:5:0:NEW 14 Mar 2020) 


\section{Table 1 (on next page)}

Table 1: The DREB gene family in pineapple 
Table 1 The DREB gene family in pineapple

\begin{tabular}{|c|c|c|c|c|c|c|c|c|}
\hline Gene ID & Gene Name & Chromosomal localization & & $\begin{array}{c}\text { Amino } \\
\text { acids } \\
\text { length (aa) }\end{array}$ & $\begin{array}{c}\text { Gene } \\
\text { length } \\
\text { (bp) }\end{array}$ & $\begin{array}{l}\text { CDS length } \\
\text { (bp) }\end{array}$ & $\begin{array}{l}\text { Isoelectric } \\
\text { points (pI) }\end{array}$ & $\begin{array}{c}\text { Molecular } \\
\text { weights (Mw) }\end{array}$ \\
\hline Aco000059 & AcoDREB04 & LG12:5065638-5067899 & 12 & 315 & 2262 & 948 & 4.91 & 33745.45 \\
\hline Aco001190 & AcoDREB16 & LG02:13530546-13531451 & 2 & 301 & 906 & 906 & 5.66 & 33079.93 \\
\hline Aco001600 & AcoDREB05 & LG18:9400576-9404316 & 18 & 341 & 3741 & 1026 & 5.05 & 38147.27 \\
\hline Aco002673 & AcoDREB11 & LG06:10539056-10539706 & 6 & 216 & 651 & 651 & 5.22 & 22927.24 \\
\hline Aco002824 & AcoDREB 17 & LG06:11885237-11886334 & 6 & 365 & 1098 & 1098 & 5.63 & 38918.03 \\
\hline Aco003376 & AcoDREB12 & LG17:2435249-2435743 & 17 & 164 & 495 & 495 & 5.79 & 18210.66 \\
\hline Aco006004 & AcoDREB07 & LG16:9780663-9781136 & 16 & 157 & 474 & 474 & 9.68 & 16405.64 \\
\hline Aco007650 & AcoDREB 18 & LG08:962022-963979 & 8 & 373 & 1958 & 1122 & 9.07 & 40044.4 \\
\hline Aco008968 & AcoDREB01 & LG09:12532806-12533489 & 9 & 227 & 684 & 684 & 6.9 & 24126.78 \\
\hline Aco009985 & AcoDREB08 & LG10:1992629-1993102 & 10 & 157 & 474 & 474 & 9.68 & 16405.64 \\
\hline Aco0 010173 & AcoDREB06 & LG25:3102765-3103427 & 25 & 220 & 663 & 663 & 5.24 & 24212.82 \\
\hline Aco012243 & AcoDREB13 & LG02:73387-74171 & 2 & 149 & 785 & 450 & 9.63 & 16316.44 \\
\hline Aco012835 & AcoDREB09 & LG03:15051238-15052266 & 3 & 342 & 1029 & 1029 & 8.68 & 36712.72 \\
\hline Aco0 14268 & AcoDREB19 & LG05:128578-129975 & 5 & 221 & 1398 & 666 & 8.56 & 24115.21 \\
\hline Aco015162 & AcoDREB 10 & LG05:1705173-1705958 & 5 & 261 & 786 & 786 & 4.71 & 27636.53 \\
\hline Aco016346 & AcoDREB20 & LG03:10461754-10463145 & 3 & 463 & 1392 & 1392 & 5.56 & 49311.65 \\
\hline Aco016696 & AcoDREB02 & LG17:191641-192357 & 17 & 238 & 717 & 717 & 7.66 & 26104.49 \\
\hline Aco018023 & AcoDREB14 & LG01:20359723-20360244 & 1 & 173 & 522 & 522 & 5.81 & 19023.86 \\
\hline Aco018980 & AcoDREB 15 & LG02:10499315-10499860 & 2 & 181 & 546 & 546 & 9.65 & 19006.18 \\
\hline Aco022517 & AcoDREB03 & LG22:6333171-6333920 & 22 & 249 & 750 & 750 & 4.98 & 25951.31 \\
\hline
\end{tabular}


Figure 1

Figure 1: Locations of ACODREB genes on the pineapple chromosomes.

The chromosome number is indicated above each bar and the length of the bar represents the size of the chromosome in pineapple. Gene start point is shown on chromosome. The figure was generated using MapChart.

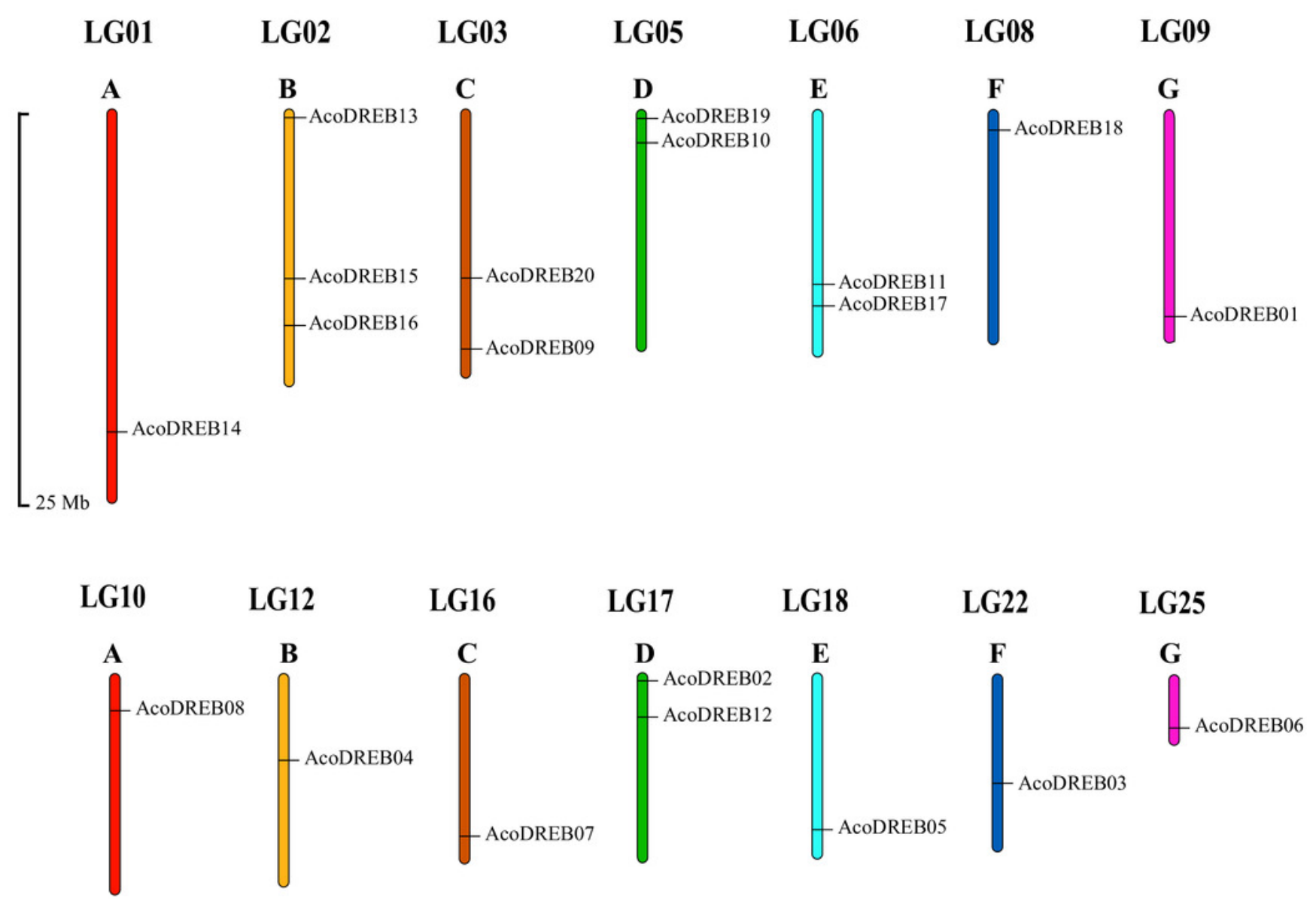




\section{Figure 2}

Figure 2: Multiple sequence alignment of the AP2 domain of AcoDREB proteins.

The alignment was performed using the DNAMAN. Conserved V14, E19, YRG and RAYD motifs are highlighted by the asterisks and lines.

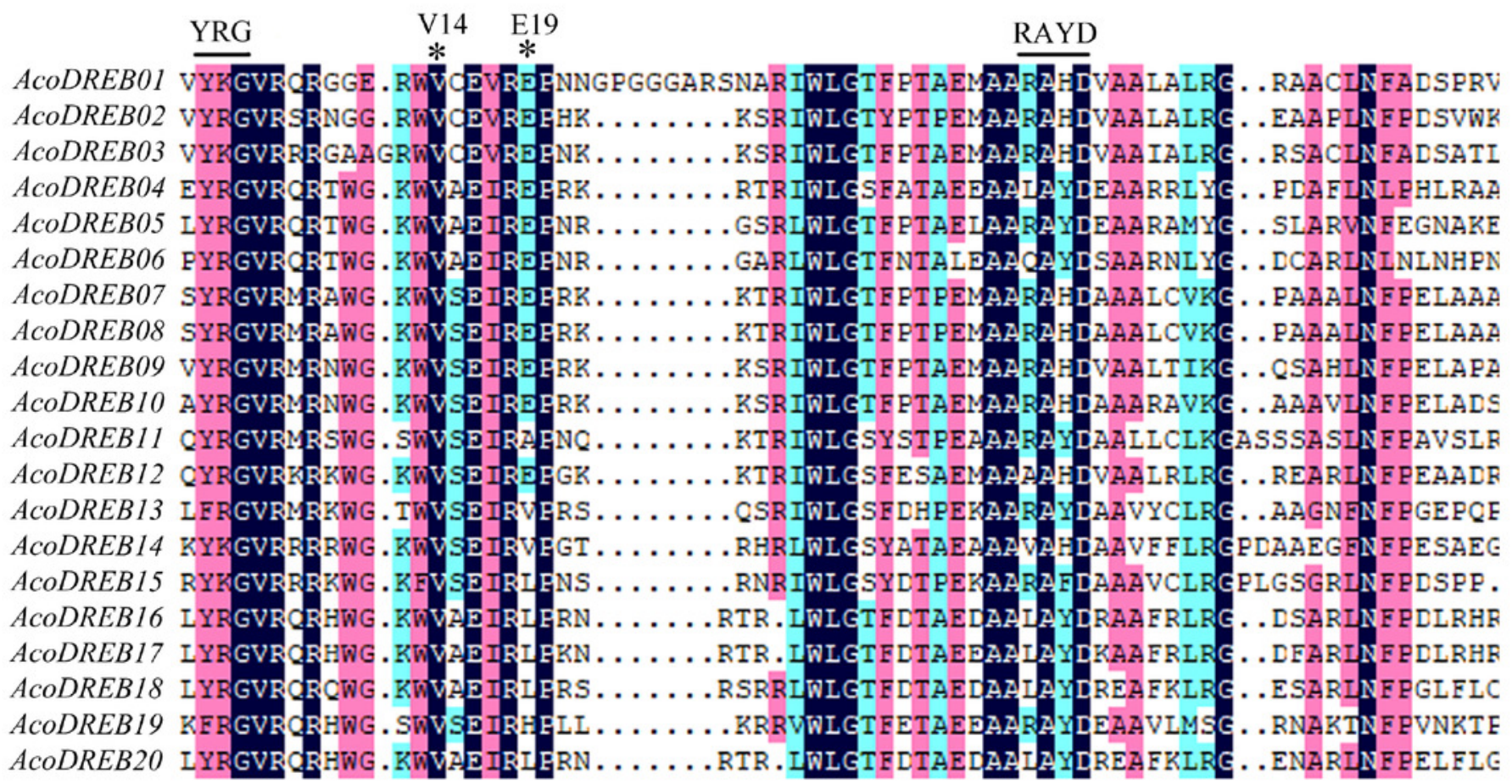


Figure 3

Figure 3: Phylogenetic analysis of DREB proteins in pineapple (Aco), Arabidopsis, and rice.

The proteins are classified into five groups: I, II, III, IV and V. Classification of Arabidopsis by Sakuma et al. (2002) is indicated in parentheses. Different individual subfamilies were shown by different color. 


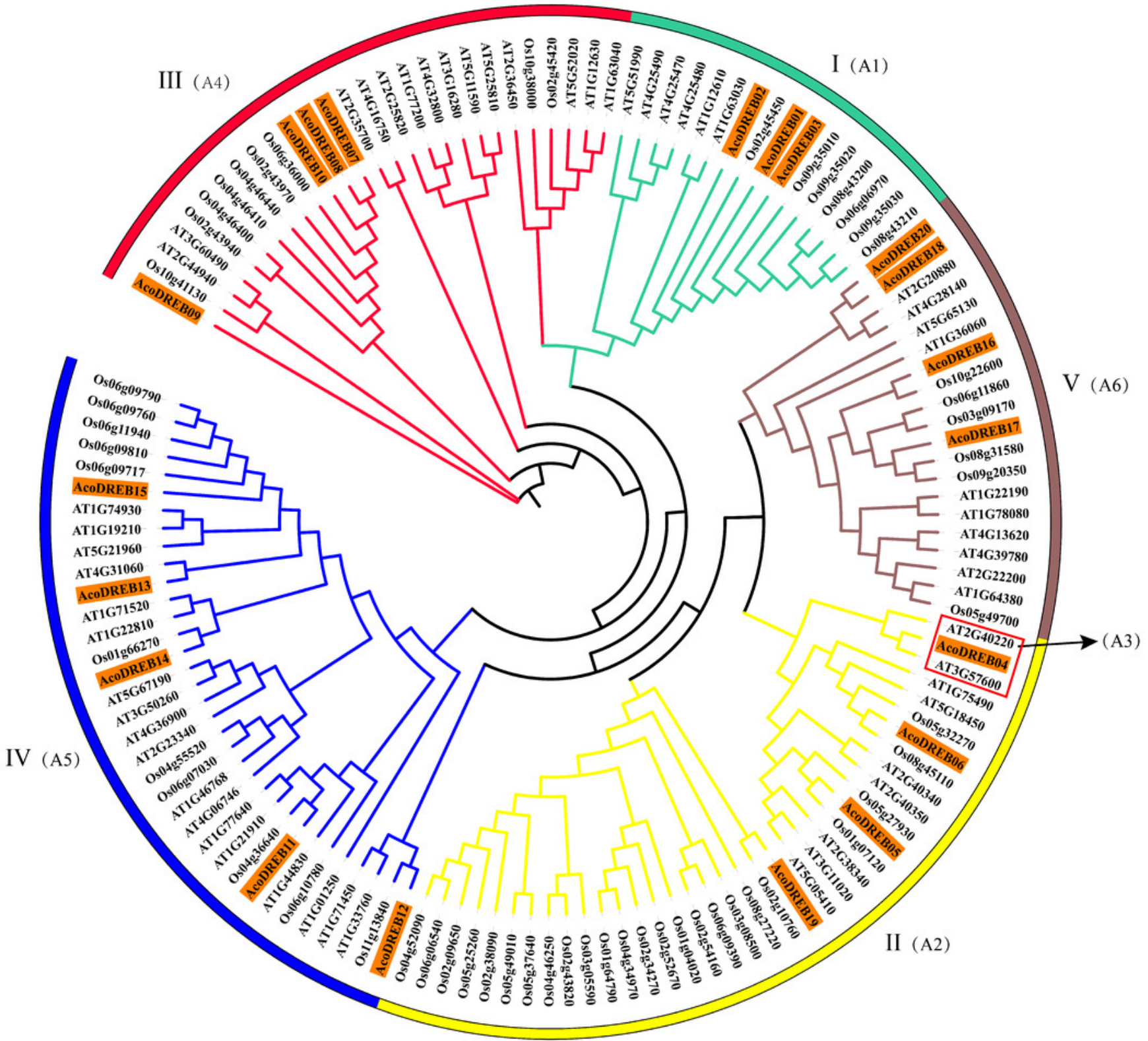




\section{Figure 4}

Figure 4: Predicted cis-elements in AcoDREB promoter regions.

Promoter sequences (-2000 bp) of 20 AcoDREB were analyzed by PlantCARE. The upstream length to the translation start site can be inferred according to the scale at the bottom. 
AcoDREB01

AcoDREB02

AcoDREB03

AcoDREB04

AcoDREB05

AcoDREB06

AcoDREB07

AcoDREB08

AcoDREB09

AcoDREB10

AcoDREB11

AcoDREB12

AcoDREB13

AcoDREB14

AcoDREB15

AcoDREB16

AcoDREB17

AcoDREB18

AcoDREB19

AcoDREB20

\section{5 '}

$-2000$

Dehydration responsive

Drought-inducibility
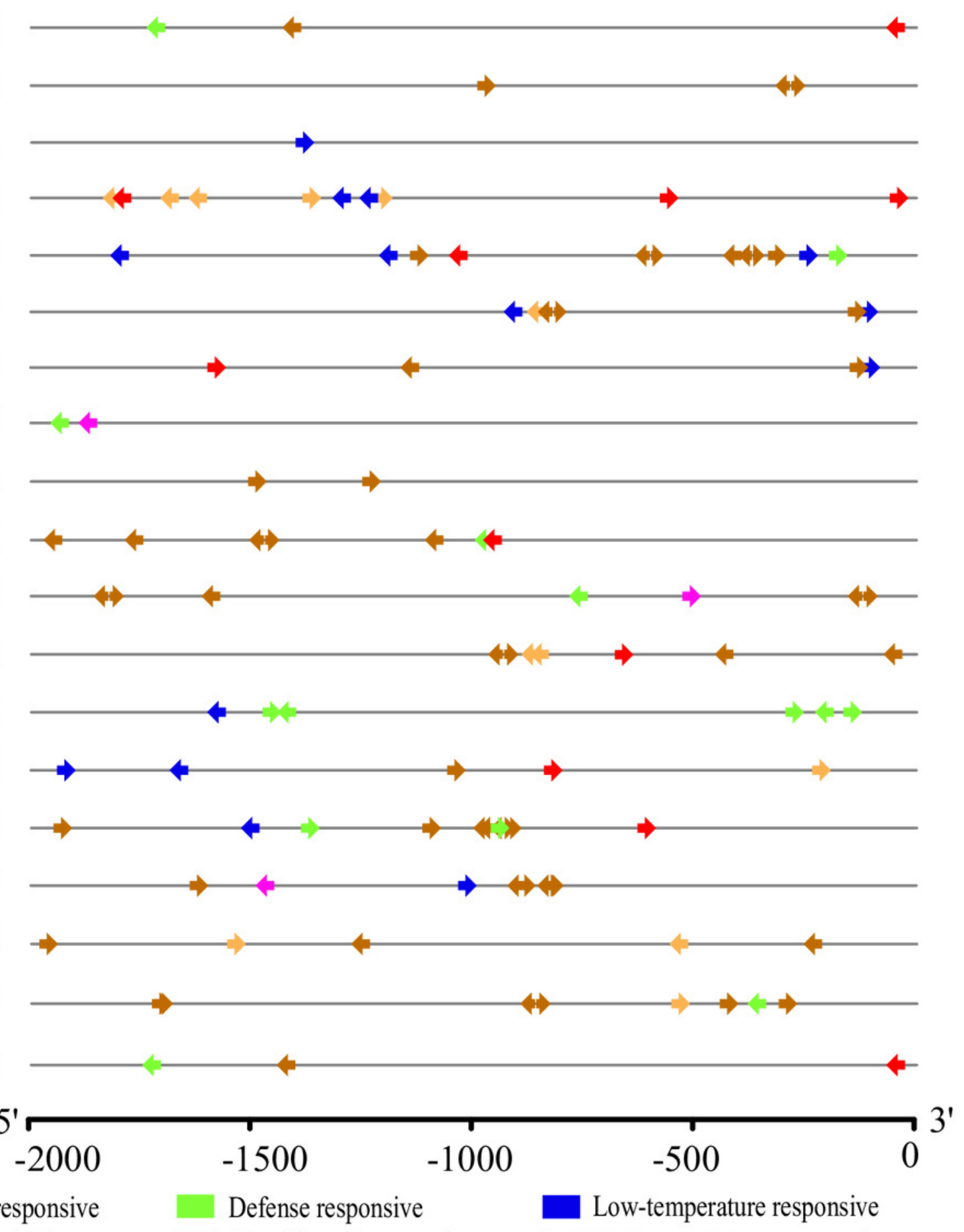

Abscisic acid responsive Defense and stress responsiveness 


\section{Figure 5}

Figure 5 : Exon-intron organization of ACODREB genes.

Black bars indicates exon (CDS), Gray bars indicated UTR while plain lines showing introns.

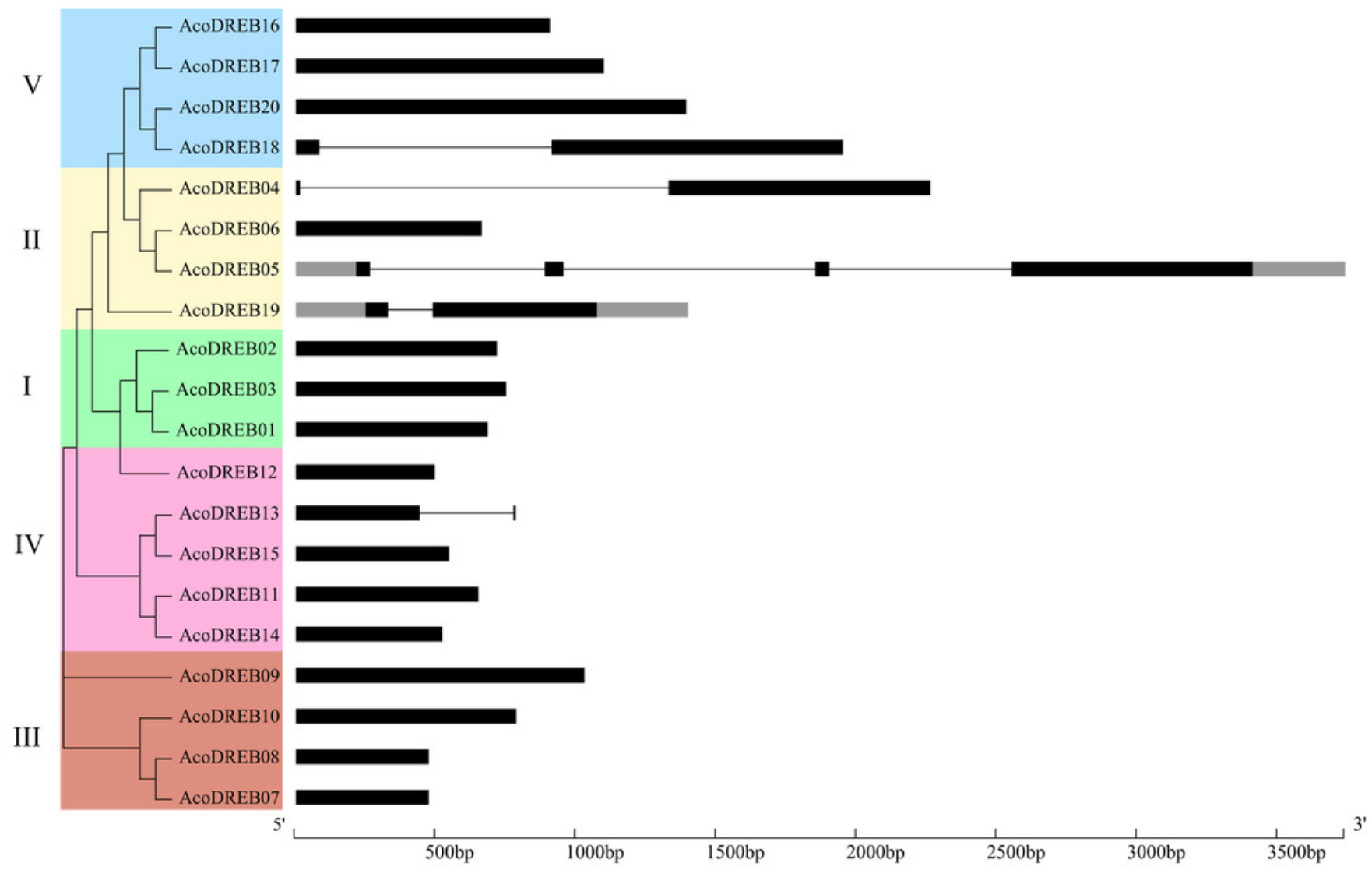

Legend:

- CDS UTR Intron 
Figure 6

Figure 6: The conserved motifs of the predicted AcoDREB proteins.

The conserved motifs in the AcoDREB proteins were identified with MEME software. Grey lines denote the non-conserved sequences, and each motif is indicated by a colored box numbered on the right. The length of motifs in each protein was presented proportionally.

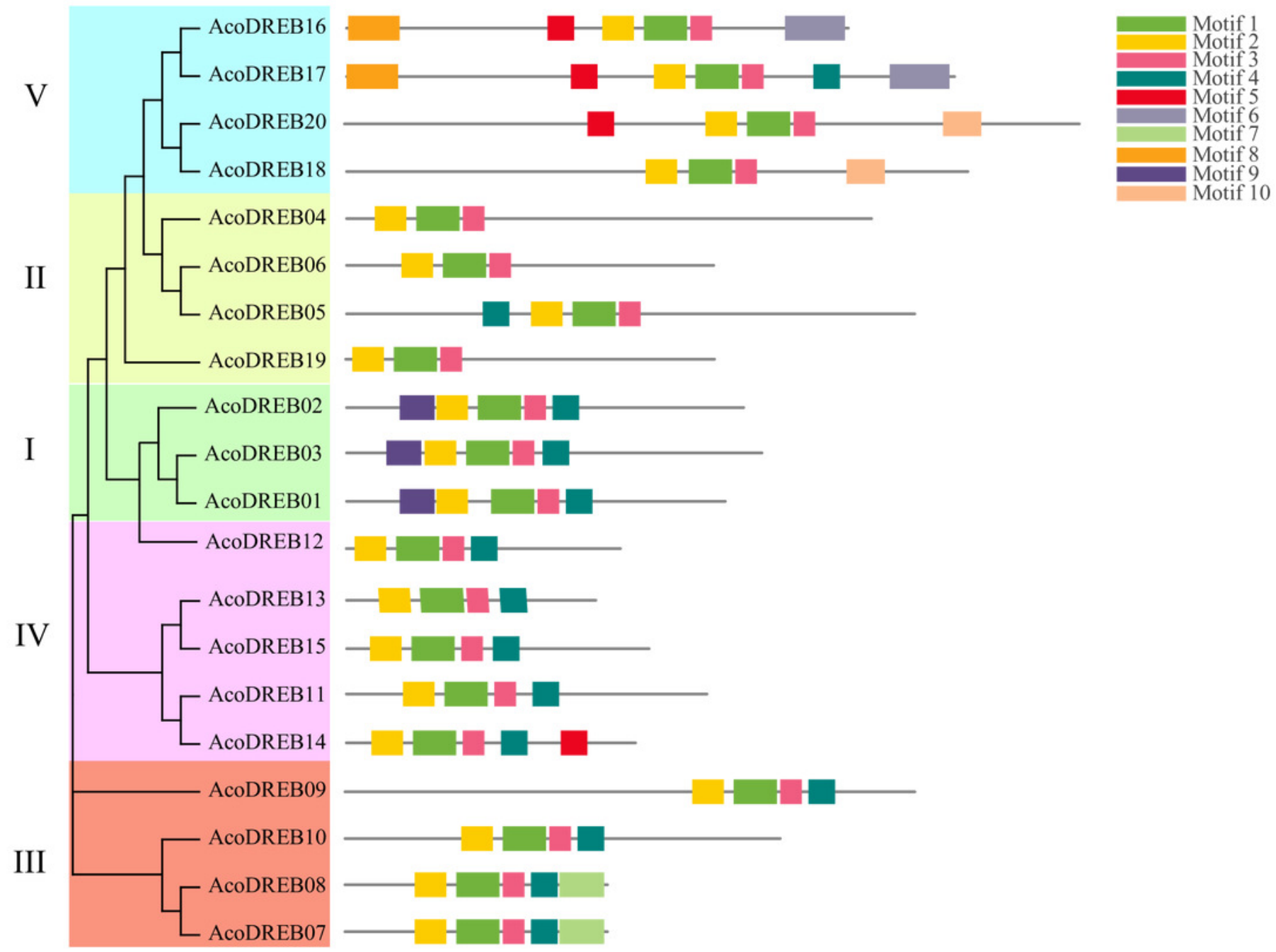




\section{Figure 7}

Figure 7: Heatmap showing the expression levels of AcoDREB genes in different pineapple tissues.

RNA-Seq expression level can be understood using the givenscale and roman numbers on right-side shows clusters based on gene expression. $\mathrm{O}, \mathrm{S}$ and $\mathrm{G}$ represent ovule, stamen and gynoecium, respectively.

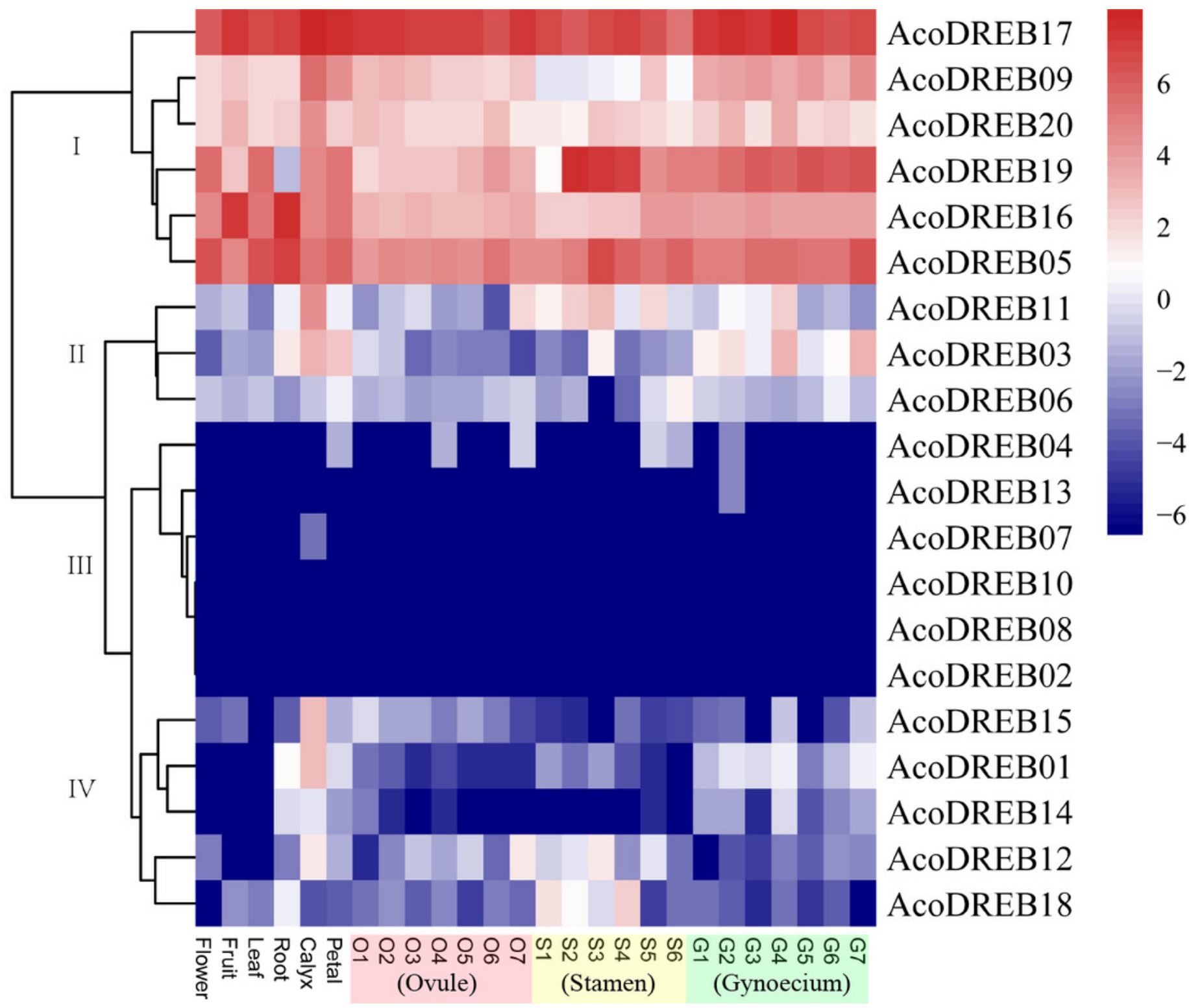




\section{Figure 8}

Figure 8: The expression profiles of AcoDREB genes in nine tissues validated by qRTPCR.

Validation of 16 genes at nine different tissues through qRT-PCR. Heat-map was constructed from relative gene expression in different tissues (qRT-PCR) data.

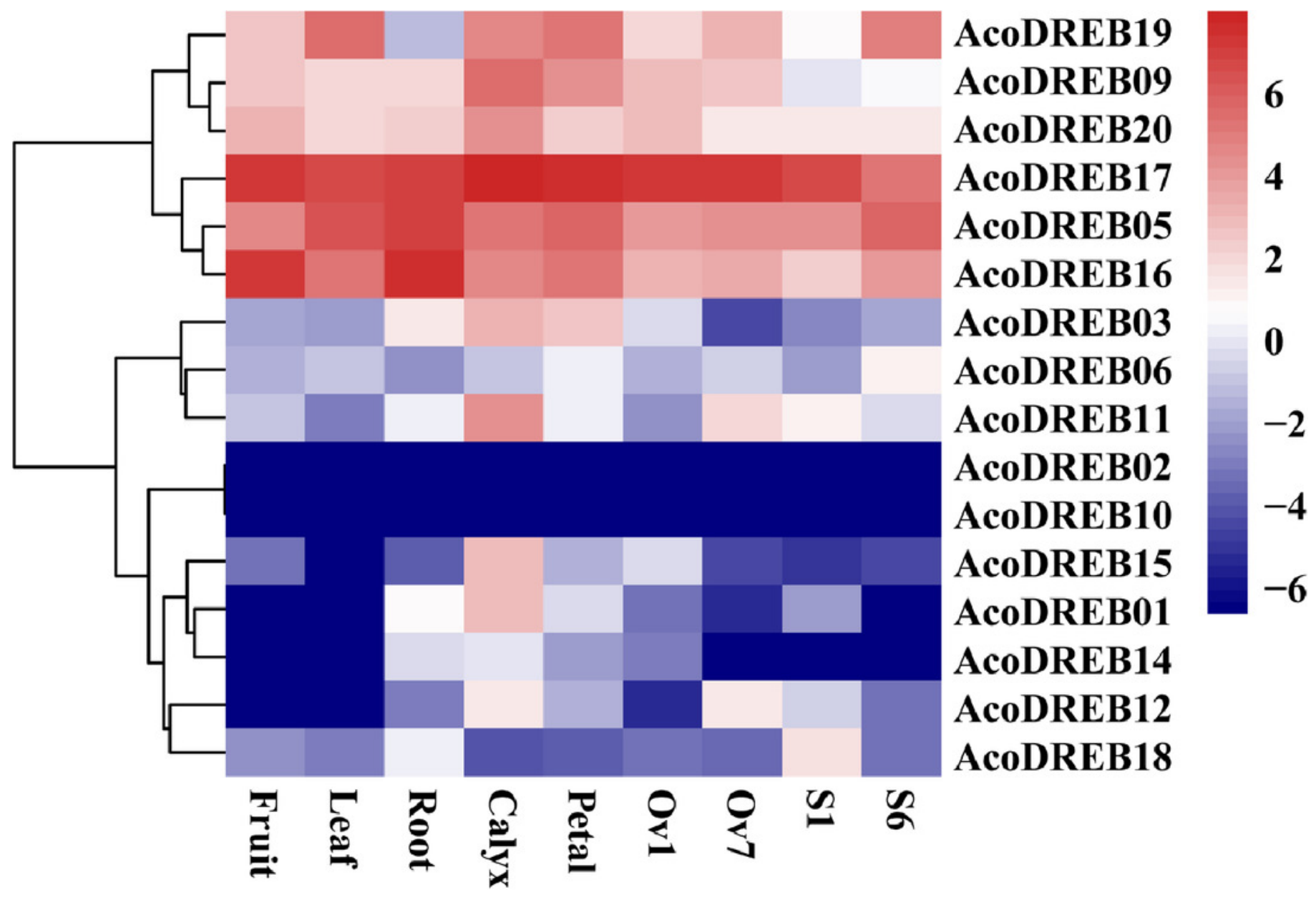


Figure 9

Figure 9: qRT-PCR expression analysis of eight selected AcoDREB genes in response to different abiotic stress treatments.

A-H high salt (150 mM NaCl); I-P drought (350 Mm Mannitol); Q-X chilling, exposure to $4{ }^{\circ} \mathrm{C}$; Y-FF high temperature, exposure to $45^{\circ} \mathrm{C}$. Mean expression value was calculated from 3 independent replicates. Error bars indicate the standard deviation. Data are presented as mean \pm standard deviation (SD). Asterisks on top of the bars indicating statistically significant differences between the stress and counterpart controls $(* P<0.05, * * P<0.01)$.
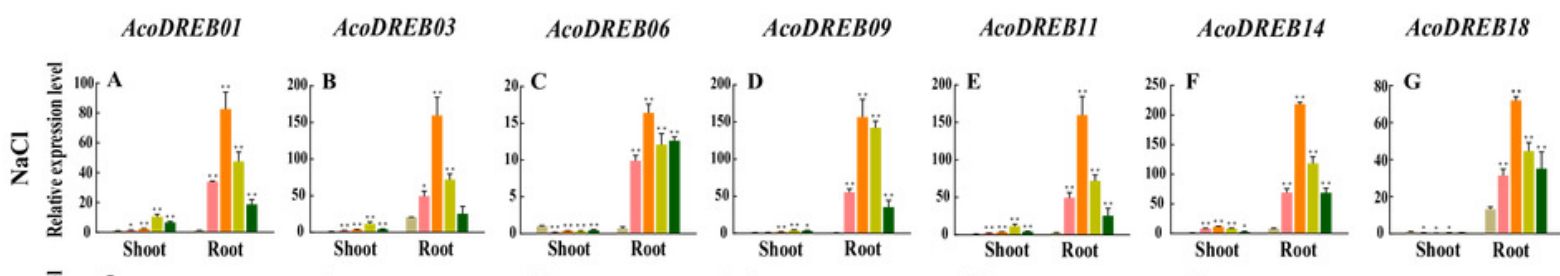

AcoDREB19
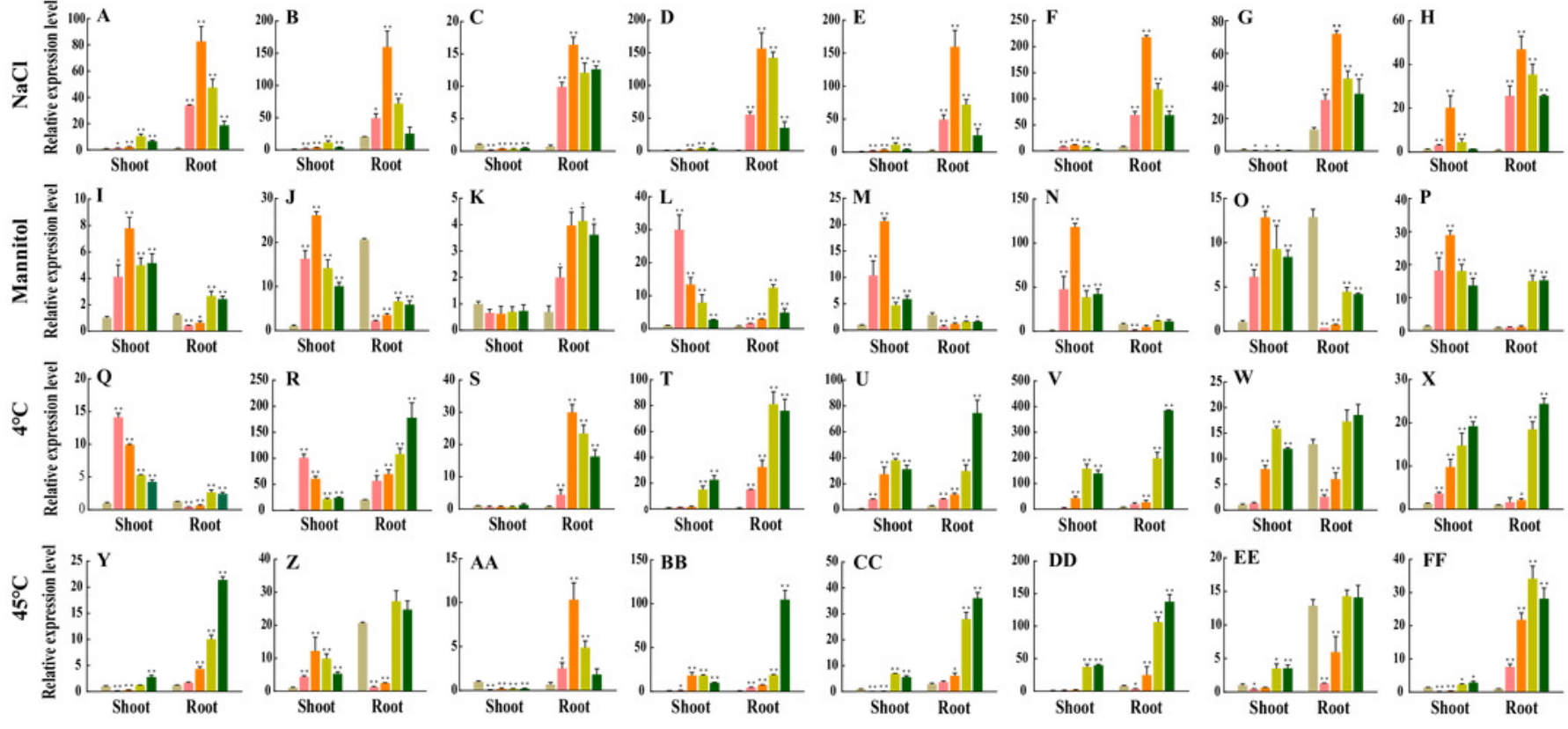

oh

$12 \mathrm{~h} \square 24 \mathrm{~h}$ 